\title{
Inverse-Compton emission from clusters of galaxies: Predictions for ASTRO-H ${ }^{\star}$
}

\author{
Richard Bartels, Fabio Zandanel, and Shin'ichiro Ando
}

GRAPPA Institute, University of Amsterdam, 1098 XH Amsterdam, The Netherlands

e-mail: r.t.bartels@uva.nl

Received 27 January 2015 / Accepted 8 June 2015

\begin{abstract}
The intra-cluster medium of several galaxy clusters hosts large-scale regions of diffuse synchrotron radio emission, known as radio halos and relics, which demonstrates the presence of magnetic fields and relativistic electrons in clusters. These relativistic electrons should also emit X-rays through inverse-Compton scattering off of cosmic microwave background photons. The detection of such a non-thermal X-ray component, together with the radio measurement, would permit the magnetic field to be clearly separated from the relativistic electron distribution because the inverse-Compton emission is independent of the magnetic field in the cluster. However, non-thermal X-rays have not been conclusively detected from any cluster of galaxies so far. In this paper, for the first time, we model the synchrotron and inverse-Compton emission of all clusters hosting radio halos and relics for which the spectral index can be determined. We provide constraints on the volume-average magnetic field by comparing with current X-ray measurements. We then estimate the maximum volume-average magnetic field that will allow detection of inverse-Compton hard X-rays by the ASTRO-H satellite. We find that several clusters are good targets for ASTRO-H to detect their inverse-Compton emission, in particular for what corresponds to radio relics, so we propose a list of promising targets for which ASTRO-H can test $\geq 1 \mu \mathrm{G}$ magnetic fields. We conclude that the already operating NuSTAR and the soon-to-be-launched ASTRO-H definitely have the potential of shedding light on the long-sought non-thermal hard-X-ray emission in clusters of galaxies.
\end{abstract}

Key words. galaxies: clusters: intracluster medium - radiation mechanisms: non-thermal - radio continuum: general X-rays: galaxies: clusters - magnetic fields

\section{Introduction}

The observation of diffuse synchrotron emission in clusters of galaxies proves that there are magnetic fields and relativistic electrons in the intra-cluster medium (ICM). This diffuse radio emission is observationally classified into two phenomena: peripheral radio relics that show irregular morphology and appear to trace merger and accretion shocks, and radio halos centred on clusters that show a regular morphology resembling that of the thermal X-ray emission (e.g., Feretti et al. 2012).

The electrons generating the observed radio emission can also produce X-rays by inverse-Compton (IC) scattering off of cosmic microwave background (CMB) photons. IC emission has been searched for extensively in the past, with a few claims of detection (see, e.g., Rephaeli \& Gruber 2002; Fusco-Femiano et al. 2004; Rephaeli et al. 2006, 2008; Eckert et al. 2008). However, more recent observations did not confirm most of the earlier claims (Molendi \& Gastaldello 2009; Ajello et al. 2009, 2010; Wik et al. 2012, 2014; Ota 2012; Ota et al. 2014; Gastaldello et al. 2015) and IC emission from clusters remains elusive.

The dominant emission in clusters of galaxies is the thermal bremsstrahlung from the ICM. Since this falls quickly above $\sim 10 \mathrm{keV}$, hard X-rays (HXRs) offer the best prospect for detecting IC emission from clusters. In coming years, nextgeneration X-ray satellites will increase the chances. Especially, ASTRO-H (Takahashi et al. 2012, 2014; Kitayama et al. 2014),

\footnotetext{
$\star$ Appendix A is available in electronic form at http://www . aanda.org
}

to be launched this year (2015), and the recently launched NuSTAR (Harrison et al. 2013) are excellent instruments for probing IC emission in HXRs.

In this work, we model, for the first time, the synchrotron and IC emission of all galaxy clusters hosting radio halos and relics for which the spectral index can be determined. The radioemitting electrons in clusters can have different origins (see, e.g., Brunetti \& Jones 2014, for a review). Our approach is phenomenological, we do not make any assumption about the injection and acceleration history of the relativistic electrons, and we only assume that the same electron distribution that generates the observed synchrotron radio emission also IC scatters off of the $\mathrm{CMB}$ photons. We provide detailed predictions for the ASTRO-H satellite and compare with current X-ray observations, where available. In particular, we estimate cap values for the volume-averaged magnetic field below which an IC signal would be detectable by ASTRO-H.

The detection of IC emission from clusters of galaxies is crucial for breaking the degeneracy in the determination of the electron distribution and magnetic field value in clusters. Because the synchrotron radio emission depends on both, while the IC emission is independent of the cluster's magnetic field, the detection of the latter in HXR is important. This will shed new light not only on non-thermal emission in clusters, but also on the impact of non-thermal phenomena on the thermal content of galaxy clusters, which is fundamental for robustly using galaxy clusters for cosmological studies (Voit 2005).

This paper is organised as follows. We introduce our sample of radio halos and relics in Sect. 2 and describe how we treat the 
relativistic electrons in Sect. 3. In Sect. 4, we briefly discuss how the non-thermal synchrotron and IC emission is modelled, while in Sect. 5, we discuss the considered background emissions. We describe the main characteristics of the ASTRO-H satellite that are important for this work in Sect. 6, and explain the procedures adopted for determining the IC detectability by ASTRO-H in Sect. 7. Finally, we present all results in Sect. 8 and our conclusions in Sect. 9. We work in cgs units and adopt a cosmological model with $\Omega_{\mathrm{m}}=0.27, \Omega_{\Lambda}=0.73$, and $H_{0}=70 \mathrm{~km} \mathrm{~s}^{-1} \mathrm{Mpc}^{-1}$.

\section{Galaxy cluster sample}

We have analysed the radio halos and relics from the September2011-Halo and September2011-Relic collections of Feretti et al. (2012). We limited our final sample to objects that have at least two radio measurements at different wavelengths in order to be able to determine the corresponding photon spectral index $\alpha$ and reduce the degeneracy in our modelling. There are a few sources for which the spectral index was estimated from neighbouring wavelengths, making the estimate subject to uncertainty. These sources were therefore excluded from our sample (A545 halo, A115, and A548 relics). Additionally, we searched the literature for radio data published after Feretti et al. (2012), which led to including the Toothbrush (1RXS J0603.3+4214), A3376, A3411, the A3667 south-eastern relic, El-Gordo (ACTCL J0102-4915), MACSJ1149.5+2223, MACSJ1752.0+4440, PLCK G171.9-40.7, and ZwCl2341.1+0000 in our final sample.

We excluded from the present analysis radio mini-halos hosted in cool-core clusters because the magnetic field estimates in the centre of these environments are high, up to $\sim 10 \mu \mathrm{G}$ (Clarke 2004; Enßlin \& Vogt 2006), implying very low relativistic electron densities for which it would be extremely difficult to aim for an IC detection ${ }^{1}$. Tables 1 and 2 contain the information about our sample of radio halos and relics, respectively, including current X-ray upper limits where available. A note on the latter: most of the current HXR upper limits come from observations pointed at the centre of clusters. There are a just few works dedicated to radio relics, but in most cases, these HXR upper limits contain contributions from both halo and relic regions.

\section{The electron population distribution}

As anticipated above, we take a phenomenological approach to the radio-emitting electrons. We assume a power-law distribution of electrons,

$N(\gamma, \theta)=K_{0} \gamma^{-(2 \alpha+1)} \frac{\sin \theta}{2}$

with $K_{0}$ the normalisation, $\gamma$ the Lorentz factor, and $\alpha$ the photon spectral index, where we consider an isotropic distribution of pitch angles $\theta$. The electron distribution is then integrated between $\gamma_{\min }$ and $\gamma_{\max }$. In some of the analysed cases, where spectral steepening is clearly observed, $\gamma_{\min }$ and $\gamma_{\max }$ can be determined from the radio data. However, more often there is no evidence of any such feature, in which case the cutoffs are fixed to theoretically motivated values.

The low-energy cutoff is estimated from the lifetime of relativistic electrons in the ICM. At low energies $\left(\gamma \lesssim 10^{2}\right)$, Coulomb cooling dominates, whereas at high energies IC cooling dominates (Rephaeli 1979; Sarazin 1999). For typical values

\footnotetext{
1 See, however, Eckert et al. (2008), Pérez-Torres et al. (2009), and Murgia et al. (2010) for the case of the Ophiuchus galaxy cluster, hosting a radio mini-halo, for which the detection of non-thermal HXR emission has been claimed.
}

in the centre of clusters, where there is a thermal electron number density of $n_{\mathrm{e}} \sim 10^{-3} \mathrm{~cm}^{-3}$ and $B=1 \mu \mathrm{G}$, this yields a maximum lifetime of $\gamma \approx 300$. However, outskirts of the cluster are characterised by lower ICM densities, which implies $\gamma \approx 200$. Therefore, the low-energy cutoff is fixed to $\gamma_{\min }=300$ for halos and to 200 for relics. The high-energy cutoff $\gamma_{\max }$ is more arbitrary given that we do not make assumptions on the injection and acceleration history of the radio-emitting electrons. If no spectral steepening at high radio frequencies is observed, it is fixed to $\gamma_{\max }=2 \times 10^{5}$, corresponding to electron energies of $100 \mathrm{GeV}$. At any rate, as we also show in Sect. 8, our conclusions are not affected by changing $\gamma_{\min }$ and/or $\gamma_{\max }$, unless, of course, spectral steepening is observed.

\section{Radiative processes}

In this work, we assume that the magnetic field is tangled on much smaller scales than the observed emitting volume, therefore adopting an isotropic distribution of magnetic field orientations. Such a distribution is motivated by Faraday rotation (FR) measurements in clusters, which indicate that the coherence scales of magnetic fields are of the order of $10 \mathrm{kpc}$, which is much smaller than the typical Mpc-size of radio halos and relics (Carilli \& Taylor 2002; Murgia et al. 2004, 2010). The synchrotron flux density is then given by

$S_{\mathrm{SYNC}}=\frac{V}{4 \pi D_{\mathrm{L}}^{2}} \int_{\gamma_{\min }}^{\gamma_{\max }} \int_{0}^{\pi} P(v, \gamma, \theta) N(\gamma, \theta) \frac{\sin \theta}{2} \mathrm{~d} \gamma \mathrm{d} \theta$,

where $P(v, \gamma, \theta)$ is the synchrotron spectrum emitted by a single electron (Blumenthal \& Gould 1970), $V$ is the emitting volume, and $D_{\mathrm{L}}$ is the luminosity distance. The factor $\sin \theta / 2$ is due to our assumption about the magnetic field distribution.

The presence of this factor leads to slightly higher estimates of the magnetic field with respect to assuming coherent magnetic fields on large scales (e.g., Wik et al. 2009; Ajello et al. 2009, 2010). This assumption of tangled magnetic fields releases part of the tension between the typically high FR measurements and equipartition estimates of the magnetic field, on the one hand, and claimed IC detections, on the other. Indeed, there are several uncertainties that affect magnetic field estimates from IC emission, and therefore the comparison with results from FR measurements. While some of these sources of uncertainties will be discussed in more detail in the next sections, we remind the reader to look at, for example, Petrosian (2001), Brunetti (2003), and Bonafede et al. (2010) for extensive discussions.

Low-energy photons scattering off of relativistic electrons can produce X-rays through IC emission. We assume that all incoming photons are from the $\mathrm{CMB}^{2}$. While starlight at the very centre of galaxy clusters can be as important as the CMB, this is not true when considering larger areas, such as the ones corresponding to radio halos or the clusters' outskirts where radio relics lie (see, e.g., Pinzke et al. 2011). The IC flux density is then given by

$S_{\text {IC }}=\frac{V}{4 \pi D_{\mathrm{L}}^{2}} \int_{0}^{\infty} \int_{\gamma_{\min }}^{\gamma_{\max }} N(\gamma) F_{\mathrm{IC}}\left(\epsilon, \gamma, \epsilon_{1}\right) \mathrm{d} \gamma \mathrm{d} \epsilon$,

where $\epsilon$ is the incoming photon energy, $\epsilon_{1}$ is the outgoing photon energy, and $F_{\mathrm{IC}}$ is the IC kernel function (Blumenthal \& Gould 1970) for the Thomson limit, which is valid for the energy range of interest here. Here, $N(\gamma)=\int_{0}^{\pi} N(\gamma, \theta) \mathrm{d} \theta$.

\footnotetext{
2 Synchrotron-self Comptonisation is also possible (e.g., Ensslin \& Sunyaev 2002).
} 
Table 1. Radio halo sample.

\begin{tabular}{|c|c|c|c|c|c|c|c|c|c|c|}
\hline Cluster & $z$ & Size & $F_{\mathrm{X}, \mathrm{UL}}$ & $\Delta E_{\mathrm{X}, \mathrm{UL}}$ & $\Gamma$ & APEC normalisation & $k T$ & Ref. 1 & Ref. 2 & Ref. 3 \\
\hline Bullet & 0.30 & 19 & 1.10 & $50-100$ & 1.9 & $7.99 \times 10^{-3}$ & 11.7 & 1,2 & 38 & 45 \\
\hline A0520 & 0.20 & 25 & & & & $1.96 \times 10^{-3}$ & 4.4 & 3 & & 45 \\
\hline A0521 & 0.25 & 20 & & & & $2.58 \times 10^{-3}$ & 4.9 & $4,5,6$ & & 45 \\
\hline A0665 & 0.18 & 79 & 4.20 & $0.6-7$ & 1.6 & $8.48 \times 10^{-3}$ & 6.8 & 7 & 39 & 45 \\
\hline A0697 & 0.28 & 20 & & & & $1.37 \times 10^{-1}$ & 8.8 & 6,8 & & 45 \\
\hline A0754 & 0.05 & 57 & 6.50 & $50-100$ & 2.0 & $3.73 \times 10^{-2}$ & 9.0 & 9,10 & 40 & 46 \\
\hline A1300 & 0.31 & 18 & & & & $2.78 \times 10^{-3}$ & 6.3 & 11 & & 45 \\
\hline Coma & 0.02 & 710 & 4.20 & $20-80$ & 2.0 & $2.18 \times 10^{-1}$ & 8.4 & 12 & 41 & 46 \\
\hline A1758a & 0.28 & 28 & & & & $2.53 \times 10^{-3}$ & 7.1 & 5,11 & & 45 \\
\hline A1914 & 0.17 & 30 & 1.09 & $50-100$ & 2.0 & $2.16 \times 10^{-2}$ & 10.5 & 9,13 & 44 & 46 \\
\hline A 2163 & 0.20 & 104 & 1.70 & $20-80$ & 1.5 & $3.23 \times 10^{-2}$ & 13.3 & 14,15 & 42 & 46 \\
\hline A2219 & 0.23 & 40 & & & & $9.84 \times 10^{-3}$ & 8.7 & 9,18 & & 45 \\
\hline A2255 & 0.08 & 79 & 2.73 & $20-80$ & 2.0 & $1.39 \times 10^{-2}$ & 6.9 & 19,20 & 43 & 46 \\
\hline A2256 & 0.06 & 116 & 2.41 & $50-100$ & 2.0 & $4.32 \times 10^{-2}$ & 7.5 & $21,22,23$ & 44 & 46 \\
\hline A2319 & 0.06 & 198 & 0.67 & $50-100$ & 2.0 & $9.81 \times 10^{-2}$ & 9.2 & 24,25 & 40 & 46 \\
\hline A 2744 & 0.31 & 38 & 0.40 & $0.6-7$ & 1.7 & $4.40 \times 10^{-3}$ & 7.2 & 18,26 & 39 & 45 \\
\hline A 3562 & 0.05 & 50 & 5.52 & $20-80$ & 2.0 & $7.67 \times 10^{-3}$ & 5.2 & 27,28 & 43 & 46 \\
\hline $\mathrm{CLO} 217+70^{a, b}$ & 0.07 & 78 & & & & $5.70 \times 10^{-3}$ & 3.6 & 29 & & 29 \\
\hline MACSJ0717.5+3745 & 0.55 & 12 & & & & $9.12 \times 10^{-3}$ & 11.6 & 30,31 & & 47 \\
\hline MACSJ1752.0+4440 & 0.37 & 23 & & & & $1.93 \times 10^{-3}$ & 6.7 & 32,33 & & 32,33 \\
\hline PLCK G171.9-40.7 ${ }^{a}$ & 0.27 & 8 & & & & $4.48 \times 10^{-3}$ & 10.7 & 34 & & 34 \\
\hline RXCJ1514.9-1523 $3^{a, b}$ & 0.22 & 40 & & & & $3.44 \times 10^{-3}$ & 6.4 & 35 & & 48 \\
\hline RXCJ2003.5-2323 $3^{a, b}$ & 0.32 & 20 & & & & $2.06 \times 10^{-3}$ & 6.8 & 36,37 & & 48 \\
\hline
\end{tabular}

Notes. We estimate the size (in $\operatorname{arcmin}^{2}$ ) of the halo in the sky in order to scale the ASTRO-H sensitivity correspondingly. In case of approximately spherical sources, this is conservatively estimated to be $\pi D_{\mathrm{LLS}} / 4$, with $D_{\mathrm{LLS}}$ the largest linear size. For elongated sources, this is approximated by a box (or ellipse) whose longest extent (or major axis) is $D_{\mathrm{LLS}} . F_{\mathrm{X}}$, UL is the current upper limit on the non-thermal hard X-ray flux in units of $10^{-12} \mathrm{erg} \mathrm{cm}^{-2} \mathrm{~s}^{-1}$, with $\Delta E_{\mathrm{X}}$, UL the corresponding energy band. $\Gamma$ is the literature spectral index used to derive $F_{\mathrm{X}}$, UL and then used to calculate the flux density for our analysis. The flux is defined as $f_{x}=k_{x} \int_{v_{\min }}^{v_{\max }} v_{x}^{-\Gamma} \mathrm{d} v_{x}$, where $k_{x}$ is a constant. The flux density is $s_{x}(v)=k_{x} v_{x}^{-\Gamma}$, with $\Gamma$ the spectral index of the IC component. The ICM temperature, $k T$, in $\mathrm{keV}$ is also shown. The three columns of references are for the radio data, the non-thermal hard X-ray upper limit, and for the input parameters of the APEC model, respectively. ${ }^{(a)}$ Gas density from Zandanel et al. (2014). (b) Temperature from $T-M_{500}$ relation of Mantz et al. (2010). ${ }^{(c)} M_{500}$ from $L_{X, \mathrm{ROSAT}}-M_{500}$ relation of Mantz et al. (2010).

References. (1) Liang et al. (2000); (2) Shimwell et al. (2014); (3) Vacca et al. (2014); (4) Brunetti et al. (2008); (5) Giovannini et al. (2009); (6) Macario et al. (2013); (7) Feretti et al. (2004); (8) van Weeren et al. (2011a); (9) Bacchi et al. (2003); (10) Kassim et al. (2001); (11) Giacintucci (2011); (12) Thierbach et al. (2003); (13) Komissarov \& Gubanov (1994); (14) Feretti et al. (2001); (15) Feretti et al. (2004); (16) Kempner \& Sarazin (2001); (17) Giovannini \& Feretti (2000); (18) Orru et al. (2007); (19) Feretti et al. (1997a); (20) Govoni et al. (2005); (21) Clarke \& Ensslin (2006); (22) Brentjens (2008); (23) van Weeren et al. (2012c); (24) Feretti et al. (1997b); (25) Storm et al. (2015); (26) Govoni et al. (2001); (27) Venturi et al. (2003); (28) Giacintucci et al. (2005); (29) Brown et al. (2011); (30) Bonafede et al. (2009a); (31) Pandey-Pommier et al. (2013); (32) Bonafede et al. (2012); (33) van Weeren et al. (2012a); (34) Giacintucci et al. (2013); (35) Giacintucci et al. (2011); (36) Venturi et al. (2009); (37) Giacintucci et al. (2009); (38) Wik et al. (2014); (39) Million \& Allen (2009); (40) Ajello et al. (2009); (41) Wik et al. (2011); (42) Ota et al. (2014); (43) Wik et al. (2012); (44) Ajello et al. (2010); (45) Fukazawa et al. (2004); (46) Chen et al. (2007), Pinzke et al. (2011); (47) Ma et al. (2008); (48) Piffaretti et al. (2011).

We conclude this section with an estimate of which HXR IC energies correspond to electrons that emit synchrotron radiation at a given frequency. This depends on the magnetic field strength. The average energy of an IC up-scattered photon is $\left\langle\epsilon_{1}\right\rangle=(4 / 3) \gamma^{2}\langle\epsilon\rangle \approx 8.6 \times 10^{-7} \gamma^{2} \mathrm{keV}$, where we assumed the incoming $\mathrm{CMB}$ photon to be at redshift 0 . We can use the monochromatic approximation for the synchrotron emission, which yields $(v / 1 \mathrm{GHz})=1.05 \times 10^{-9} \gamma^{2}(B / 1 \mu \mathrm{G})$. In the case of $B=1 \mu \mathrm{G}$, synchrotron radiation at $\sim 100(20) \mathrm{MHz}$ is produced by the electrons that generate IC emission at 80 (20) $\mathrm{keV}$, while for $B=5 \mu \mathrm{G}$, one finds that $\sim 480(120) \mathrm{MHz}$ radio waves correspond to $80(20) \mathrm{keV} \mathrm{X}$-rays. Therefore, lowfrequency radio observations are crucial for precisely predicting HXR IC emission, as discussed in Sect. 8, and for eventually clarifying whether our assumption of Sect. 3 regarding the electron distribution holds down to low energies.

\section{Background emission}

In this section, we discuss the main background emission for IC searches in clusters. These are the thermal ICM bremsstrahlung and the cosmic X-ray background (CXB) emission. In Sect. 7, we also introduce the instrumental background.

\subsection{Thermal bremsstrahlung from ICM}

To model the thermal bremsstrahlung emission from the ICM, we use the APEC code (Smith et al. 2001) as provided in the XSPEC software (Arnaud 1996). The input parameters are the ICM temperature (taken, for most nearby clusters, from Chen et al. 2007), the redshift $z$, the metal abundance, which we fix to 0.3 times the solar abundance for simplicity (e.g., Böhringer \& Werner 2010), and the normalisation. The latter contains information about the ICM gas distribution that, for 
Table 2. Radio relic sample.

\begin{tabular}{|c|c|c|c|c|c|c|c|c|c|c|c|}
\hline Cluster & Source & $z$ & Size & $F_{\mathrm{X}, \mathrm{UL}}$ & $\Delta E_{\mathrm{X}, \mathrm{UL}}$ & $\Gamma$ & APEC normalisation & $k T$ & Ref. 1 & Ref. 2 & Ref. 3 \\
\hline Toothbrush $^{a, c}$ & & 0.23 & 18 & & & & $6.4 \times 10^{-4}$ & 7.8 & 1 & & 49 \\
\hline A0013 & & 0.09 & 5 & & & & $3.0 \times 10^{-3}$ & 6.0 & 2 & & 50 \\
\hline A0085 & & 0.06 & 26 & 2.5 & $50-100$ & 2.0 & $5.1 \times 10^{-3}$ & 6.1 & 2 & 43 & 51 \\
\hline A0521 & & 0.25 & 9 & & & & $5.9 \times 10^{-5}$ & 4.9 & 3 & & 52 \\
\hline $\mathrm{A} 0610^{a, b, e}$ & & 0.10 & 8 & & & & $1.3 \times 10^{-4}$ & 2.4 & 4 & & 54 \\
\hline $\mathrm{AS} 753^{a}$ & & 0.01 & 318 & & & & $4.2 \times 10^{-4}$ & 2.5 & 5 & & 5 \\
\hline A0754 & & 0.05 & 100 & & & & $9.0 \times 10^{-3}$ & 9.0 & 6,7 & & 6 \\
\hline $\mathrm{A} 0781^{a, b}$ & & 0.30 & 6 & & & & $3.4 \times 10^{-5}$ & 7.6 & 8 & & 55 \\
\hline \multirow[t]{2}{*}{ A1240 } & $\mathrm{N}$ & 0.16 & 12 & & & & $3.7 \times 10^{-6}$ & 6.0 & 9 & & 56 \\
\hline & $S$ & 0.16 & 24 & & & & $1.5 \times 10^{-6}$ & & 9 & & \\
\hline A1300 & & 0.31 & 6 & & & & $1.8 \times 10^{-5}$ & 6.3 & 10,11 & & 52 \\
\hline A1367 & & 0.02 & 52 & 8.2 & $20-80$ & 2.0 & $2.7 \times 10^{-4}$ & 3.6 & 12 & 63 & 51 \\
\hline $\mathrm{A} 1612^{a, b}$ & & 0.18 & 8 & & & & $3.3 \times 10^{-6}$ & 4.9 & 13 & & 57 \\
\hline Coma & & 0.02 & 400 & 0.3 & $0.3-10$ & 2.2 & $8.5 \times 10^{-4}$ & 8.4 & 14 & 45 & 51 \\
\hline A1664 & & 0.13 & 51 & & & & $6.0 \times 10^{-4}$ & 6.8 & 8 & & 27 \\
\hline $\mathrm{A} 2048^{a, b, d}$ & & 0.10 & 7 & & & & $4.0 \times 10^{-4}$ & 4.2 & 15 & & 58 \\
\hline A2061 & & 0.08 & 24 & & & & $6.6 \times 10^{-4}$ & 4.5 & 13,16 & & 59 \\
\hline A2063 & & 0.03 & 3 & 7.6 & $20-80$ & 2.0 & $3.6 \times 10^{-3}$ & 3.7 & 17 & 44 & 51 \\
\hline A 2163 & & 0.20 & 5 & & & & $1.6 \times 10^{-5}$ & 13.3 & 18,19 & & 51 \\
\hline A2255 & & 0.08 & 32 & & & & $3.2 \times 10^{-4}$ & 6.9 & 20 & & 51 \\
\hline A2256 & & 0.06 & 152 & & & & $2.3 \times 10^{-2}$ & 7.5 & $21,22,23$ & & 51 \\
\hline \multirow[t]{2}{*}{$\mathrm{A} 2345^{a, b}$} & $\mathrm{E}$ & 0.18 & 38 & & & & $2.6 \times 10^{-4}$ & 6.5 & 24 & & 53 \\
\hline & W & & 35 & & & & $8.5 \times 10^{-4}$ & & 24 & & \\
\hline $\mathrm{A} 2433^{a, b, e}$ & & 0.11 & 11 & & & & $2.7 \times 10^{-5}$ & 1.8 & 25 & & 60 \\
\hline A2744 & & 0.31 & 19 & 0.4 & $0.6-7$ & 1.7 & $6.6 \times 10^{-5}$ & 7.2 & 26,27 & 46 & 52 \\
\hline \multirow[t]{2}{*}{ A3376 } & E & 0.05 & 324 & 3.5 & $4-8$ & 2.0 & $5.4 \times 10^{-3}$ & 4.3 & 28 & 47 & 51 \\
\hline & $\mathrm{W}$ & & 122 & 1.1 & $4-8$ & 2.0 & $3.1 \times 10^{-5}$ & & 28 & 47 & \\
\hline $\mathrm{A} 3411^{a}$ & & 0.17 & 62 & & & & $3.2 \times 10^{-3}$ & 6.4 & 29 & & 29 \\
\hline \multirow[t]{2}{*}{ A3667 } & NW & 0.06 & 250 & 0.6 & $10-40$ & 1.8 & $6.6 \times 10^{-4}$ & 7.0 & $30,31,32$ & 48 & 51 \\
\hline & SE & & 175 & & & & $1.9 \times 10^{-3}$ & & 31,32 & & \\
\hline A4038 & & 0.03 & 12 & 7.4 & $20-80$ & 2.0 & $2.8 \times 10^{-4}$ & 3.2 & 2,8 & 44 & 51 \\
\hline \multirow[t]{3}{*}{ El-Gordo ${ }^{a}$} & E & 0.87 & 3 & & & & $2.7 \times 10^{-2}$ & 14.5 & 33 & & 61 \\
\hline & NW & & 3 & & & & $2.7 \times 10^{-2}$ & & 33 & & \\
\hline & SE & & 3 & & & & $2.7 \times 10^{-2}$ & & 33 & & \\
\hline Sausage $\mathrm{e}^{a, b, c}$ & & 0.19 & 18 & & & & $1.1 \times 10^{-5}$ & 5.6 & 34 & & 57 \\
\hline \multirow[t]{2}{*}{ MACSJ1149.5+2223 ${ }^{a}$} & $\mathrm{E}$ & 0.54 & 2 & & & & $2.5 \times 10^{-7}$ & 14.5 & 35 & & 35 \\
\hline & $\mathrm{W}$ & & 2 & & & & $4.8 \times 10^{-7}$ & & 35 & & \\
\hline \multirow[t]{2}{*}{ MACSJ1752.0+4440 ${ }^{a, c}$} & $\mathrm{NE}$ & 0.37 & 8 & & & & $6.3 \times 10^{-6}$ & 6.7 & 35,36 & & 35,36 \\
\hline & SW & & 3 & & & & $1.2 \times 10^{-5}$ & & 35,36 & & \\
\hline \multirow[t]{2}{*}{ PLCK G287.0+32.9 $9^{a, c}$} & $\mathrm{~N}$ & 0.39 & 7 & & & & $7.4 \times 10^{-5}$ & 12.9 & 37 & & 57 \\
\hline & $\mathrm{S}$ & 0.39 & 6 & & & & $5.2 \times 10^{-7}$ & 12.9 & 37 & & \\
\hline \multirow[t]{2}{*}{ RXCJ1314.4-2515 } & $\mathrm{E}$ & 0.25 & 8 & & & & $3.5 \times 10^{-6}$ & 7.2 & 38,39 & & 57 \\
\hline & W & & 15 & & & & $2.3 \times 10^{-4}$ & & 38,39 & & \\
\hline $\mathrm{ZwCl} 0008.8-5215^{a, b}$ & $\mathrm{E}$ & 0.10 & 36 & & & & $1.3 \times 10^{-4}$ & 5.0 & 40 & & 53 \\
\hline \multirow[t]{2}{*}{$\mathrm{ZwCl} 2341.1+0000^{a}$} & $\mathrm{~N}$ & 0.27 & 4 & & & & $8.7 \times 10^{-5}$ & 9.3 & 41,42 & & 62 \\
\hline & $\mathrm{S}$ & & 20 & & & & $1.9 \times 10^{-4}$ & & 41,42 & & \\
\hline
\end{tabular}

Notes. Columns are as in Table 1, with the addition of the second column, which indicates the specific relic to which we are referring. ${ }^{(a)}$ Gas density from Zandanel et al. (2014). ${ }^{(b)}$ Temperature from $T-M_{500}$ relation of Mantz et al. (2010). ${ }^{(c)} M_{500}$ from $L_{X, \mathrm{ROSAT}}-M_{500}$ relation of Mantz et al. (2010). ${ }^{(d)} M_{500}$ from $L_{X \text {,bolometric }}-M_{500}$ of Mantz et al. (2010). ${ }^{(e)} M_{500}$ scaled from $M_{200}$.

References. (1) van Weeren et al. (2012b); (2) Slee et al. (2001); (3) Macario et al. (2013); (4) Giovannini \& Feretti (2000); (5) Subrahmanyan et al. (2003); (6) Bacchi et al. (2003); (7) Kassim et al. (2001); (8) Kale \& Dwarakanath (2012); (9) Bonafede et al. (2009b); (10) Reid et al. (1999); (11) Giacintucci (2011); (12) Gavazzi \& Trinchieri (1983); (13) van Weeren et al. (2011a); (14) Thierbach et al. (2003); (15) van Weeren et al. (2011c); (16) Kempner \& Sarazin (2001); (17) Komissarov \& Gubanov (1994); (18) Feretti et al. (2001); (19) Feretti et al. (2004); (20) Feretti et al. (1997a); (21) Clarke \& Ensslin (2006); (22) Brentjens (2008); (23) van Weeren et al. (2012c); (24) Bonafede et al. (2009b); (25) Cohen \& Clarke (2011); (26) Orru et al. (2007); (27) Govoni et al. (2001); (28) Kale et al. (2012); (29) van Weeren et al. (2013); (30) Rottgering et al. (1997); (31) Hindson et al. (2014); (32) Johnston-Hollitt (2004); (33) Lindner et al. (2014); (34) Stroe et al. (2014); (35) Bonafede et al. (2012); (36) van Weeren et al. (2012a); (37) Bagchi et al. (2011); (38) Feretti et al. (2005); (39) Venturi et al. (2007); (40) van Weeren et al. (2011b); (41) van Weeren et al. (2009); (42) Bagchi et al. (2002); (43) Ajello et al. (2010); (44) Wik et al. (2012); (45) Feretti \& Neumann (2006); (46) Million \& Allen (2009); (47) Kawano et al. (2009); (48) Nakazawa et al. (2009); (49) Ogrean et al. (2013); (50) Juett et al. (2008); (51) Chen et al. (2007), Pinzke et al. (2011); (52) Fukazawa et al. (2004); (53) Planck Collaboration XXIX (2014); (54) Yoon et al. (2008); (55) Sehgal et al. (2008); (56) Barrena et al. (2009), Cavagnolo et al. (2009); (57) Piffaretti et al. (2011); (58) Shen et al. (2008); (59) Marini et al. (2004); (60) Popesso et al. (2007); (61) Menanteau et al. (2012); (62) Boschin et al. (2013); (63) Henriksen \& Mushotzky (2001). 
our purposes, can be approximated with a beta model (e.g., Cavaliere \& Fusco-Femiano 1976),

$n_{\mathrm{e}}(r)=\left[\sum_{i} n_{0, i}^{2}\left(1+\frac{r^{2}}{r_{c, i}^{2}}\right)^{-3 \beta_{i}}\right]^{1 / 2}$,

where $n_{0}$ is the central ICM electron density, and $r_{\mathrm{c}}$ the core radius. Most clusters are described well by a single beta model $(i=1)$, whereas others are better described by a double beta model $(i=1,2)$. The values of $n_{0}, r_{\mathrm{c}}$, and $\beta$ are derived from $\mathrm{X}$-ray observations. For most nearby clusters, these values are taken from Pinzke et al. (2011).

In the few cases where we cannot find a gas density model in the literature, we adopt the phenomenological ICM model of Zandanel et al. (2014) which provides a gas density once the mass $M_{500}$ of the cluster is known. In the few cases where no mass estimate is present in the literature, we adopt the X-ray luminosity-mass relation from Mantz et al. (2010). If there is no ICM temperature available in the literature, we also use the mass-temperature relation of Mantz et al. (2010). Obviously, such approximations are a source of uncertainty when used in particular cases, so the modelling of those clusters should be taken with caution. This is also true in general for relics where the gas density profiles have been inferred from X-ray observations of the clusters' centre.

In the case of radio halos, the bremsstrahlung luminosity, $L_{\mathrm{B}}$, is obtained by integrating over the full extent of the halo, where we approximate the halo to be spherical and use the largest linear size as given in Feretti et al. (2012) as halo diameter. In the case of radio relics in the outskirts of clusters, we only integrate over the relic region as

$L_{\mathrm{B}} \sim(1-\cos \phi) \int_{R_{\mathrm{cc}}-0.5 R_{\mathrm{w}}}^{R_{\mathrm{cc}}+0.5 R_{\mathrm{w}}} n_{\mathrm{e}}(r)^{2} r^{2} \mathrm{~d} r$,

where $R_{\mathrm{cc}}$ is the distance to the cluster centre, $R_{\mathrm{w}}$ the approximate width of the relic, and $\phi=\tan ^{-1}\left[0.5 R_{\mathrm{l}} /\left(R_{\mathrm{cc}}-0.5 R_{\mathrm{W}}\right)\right]$ with $R_{1}$ the largest linear size of the relic (see Fig. 1). For relics that are classified as roundish by Feretti et al. (2012), we use $R_{\mathrm{w}}=R_{\mathrm{l}}$.

The parameters that enter the APEC model are shown in Tables 1 and 2 for all the clusters in our sample.

\subsection{Cosmic $X$-ray background}

The CXB (e.g., Gilli et al. 2007) is another important background for the case of radio relics in the outskirts of clusters, where it often outweighs the thermal ICM emission. We assume the CXB to be isotropic across the sky and model it in the 0.5-100 keV regime as follows (Gruber 1992; Comastri et al. 1995):

$13.6 E^{-1}$ for $0.5 \leq E<2 \mathrm{keV}$,

$7.877 E^{-0.29} \mathrm{e}^{-E /(41.13 \mathrm{keV})}$ for $2 \leq E<60 \mathrm{keV}$,

$1652 E^{-2}+1.754 E^{-0.7}$ for $60 \leq E \leq 100 \mathrm{keV}$,

in units of $\mathrm{keV} \mathrm{cm}^{-2} \mathrm{~s}^{-1} \mathrm{sr}^{-1} \mathrm{keV}^{-1}$ and with $E$ the photon energy in units of $\mathrm{keV}$. Below $0.5 \mathrm{keV}$, the galactic emission becomes an important source of background, but these low energies are not considered in this work.

\section{The ASTRO-H and NuSTAR satellites}

In this section, we describe the characteristics of ASTRO-H that are relevant for this work. While we provide detailed predictions

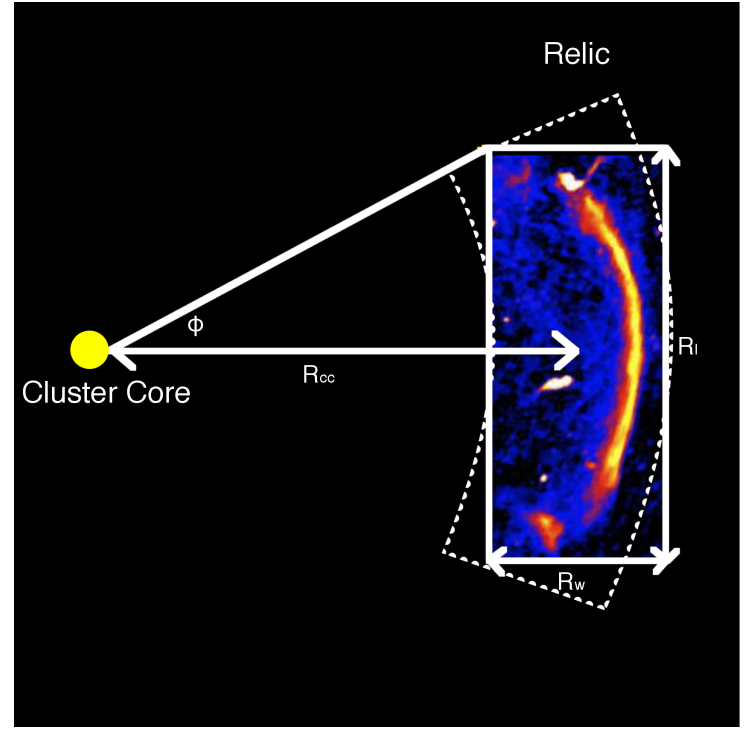

Fig. 1. The geometry used in determining the normalisation for the APEC modelling of the radio relic regions (Eq. (5)). $R_{\mathrm{cc}}$ corresponds to the distance between the relic and the cluster center as projected on the sky. $R_{\mathrm{l}}$ is the relic largest linear size and $R_{\mathrm{w}}$ is the relic width. For relics that are classified as roundish by Feretti et al. (2012) we use $R_{\mathrm{w}}=R_{\mathrm{l}}$. $\phi$ is given by $\tan ^{-1}\left(\frac{0.5 R_{\mathrm{l}}}{R_{\mathrm{cc}}-0.5 R_{\mathrm{W}}}\right)$. In result, the dashed curve is the solid angle over which we integrate. The relic image, corresponding to the so-called Sausage, is adapted from Röttgering et al. (2013).

Table 3. Properties of the SXI and HXI detectors that will be on-board ASTRO-H.

\begin{tabular}{lcc}
\hline \hline & SXI & HXI \\
\hline Energy range [keV] & $0.4-12.0$ & $5-80$ \\
Angular resolution [arcmin] & 1.3 & 1.7 \\
Field of view (FoV) [arcmin $\left.{ }^{2}\right]$ & $38 \times 38$ & $9 \times 9$ \\
Energy resolution [eV] & $150 @ 6 \mathrm{keV}$ & $<2000 @ 60 \mathrm{keV}$ \\
Effective area [cm $\left.{ }^{2}\right]$ & $360 @ 6 \mathrm{keV}$ & $300 @ 30 \mathrm{keV}$ \\
\hline
\end{tabular}

Notes. Values adopted from the ASTRO-H Quick Reference.

for the IC-emission detection by ASTRO-H, we also discuss in the same context the recently launched NuSTAR satellite.

\subsection{ASTRO-H}

ASTRO-H is a next-generation X-ray satellite that is scheduled for launch in 2015 (Takahashi et al. 2012, 2014). The instruments that are of interest for our purposes are the Hard X-ray Imager (HXI) and the Soft X-ray Imager (SXI). In Table 3, we summarise the main characteristics of these instruments.

To estimate the detectability of the non-thermal X-ray component in galaxy clusters, we make use of the sensitivity curves as published by the ASTRO-H collaboration for both point and extended sources (ASTRO-H Quick Reference ${ }^{3}$, Takahashi et al. 2010,2012 ). We adopt the sensitivity curve for $1 \mathrm{Ms}$ of observation, and scale it to other observation times as $\sqrt{\text { time. The }}$ ASTRO-H sensitivity curves are $3 \sigma$ sensitivities, and the sensitivity curve for extended sources assumes a uniform source of $1 \mathrm{deg}^{2}$.

3 http://astro-h.isas . jaxa.jp/ahqr.pdf 
We assume, for simplicity, that halos and relics are uniform sources. To scale the sensitivity to the size, $\Omega \mathrm{arcmin}^{2}$, of a given object, we proceed as follows. For sources that are smaller than the HXI field of view, we scale the point-source sensitivity as $\sqrt{\Omega / 2 \operatorname{arcmin}^{2}}$, where $2 \operatorname{arcmin}^{2}$ is roughly the pixel size. For sources that are larger, we apply a linear scaling to the $1 \mathrm{deg}^{2}$ diffuse source sensitivity as $\Omega / 3600 \operatorname{arcmin}^{2}$ (Hiroki Akamatsu, Madoka Kawaharada, priv. comm.).

\subsection{NUSTAR}

The NuSTAR satellite was launched on June 13, 2012. Its performances in HXR should be comparable to ASTRO-H (Harrison et al. 2013), with the exception that ASTRO-H will be equipped with a pre-collimator designed to mitigate stray light (Mori et al. 2012).

Recently, Wik et al. (2014) have pointed out that a possible IC signal has to be extracted from both the thermal and instrumental backgrounds, with the former dominating at low and the latter at high energies. In fact, the instrumental background is likely to dominate the count rate in the HXR. Since the instrumental background is modelled by a power law, any claim of IC detection is extremely sensitive to the applied model. While the HXI detector onboard ASTRO-H will not improve much upon NuSTAR - in fact, our predictions are approximately valid in both cases - the presence of the SXI detector will greatly improve the modelling of the thermal component in clusters over the NuSTAR results.

\section{Multi-band modelling and HXR detectability}

For any given object in Tables 1 and 2, we proceed as follows. If an upper limit exists on the non-thermal hard X-ray flux $F_{\mathrm{X}, \mathrm{UL}}$, we calculate the corresponding lower limit on the volume-average magnetic field $B_{\mathrm{V}}$. First, we fix $K_{0}$ by fitting the IC spectrum of Eq. (3) to $F_{X, U L}$. Next, we fit the synchrotron emission of Eq. (2) to the radio data ${ }^{4}$, with $B_{\mathrm{V}}$ as free parameter. Depending on whether spectral steepening at low and/or high frequencies is observed, $\gamma_{\min }$ and/or $\gamma_{\max }$ are also determined from the data (see Sect. 3). The spectral index $\alpha$ is taken from the literature, unless it returns a poor fit, in which case we determine a new spectral index. The resulting broadband spectrum provides a lower limit on the magnetic field.

We then compare the background spectra (thermal bremsstrahlung and the CXB) to the properly scaled ASTRO-H sensitivity. We use the intersection between the ASTRO-H sensitivity curve and the dominant background component, either thermal ICM or CXB, to fix a new normalisation of the electron distribution $K_{0}$ using Eq. (3). This represents the lower limit on the normalisation detectable by ASTRO-H. If no upper limit on the hard X-ray flux, $F_{X, U L}$, exists for a given object, we start from this step. Finally, we fit the synchrotron emission of Eq. (2) again to the radio data. The resulting magnetic field value is a cap on the volume-average magnetic field below which HXR IC emission would be detectable by ASTRO-H, and we call it $B_{\mathrm{dt}}$. To be clearer, HXR IC emission would be detectable by ASTRO-H if the cluster's magnetic field was lower than or equal to $B_{\mathrm{dt}}$, always meaning a volume average. We underline that this simple approach is meant to be just a first-order approximation to what can really be achieved. In

\footnotetext{
${ }^{4}$ We neglect the possible effect at high frequencies of thermal Sunyaev-Zeldovich (SZ) decrement (e.g., Enßlin 2002) which should, however, have a small impact (e.g., Brunetti et al. 2013). Anyhow, ignoring the SZ decrement implies more conservative results.
}

fact, the CXB background is well known and can be easily subtracted. We provide the result in such a case, along with the rest, in Tables 4 and 5.

The above approach leads to a rough estimate that we complement with one based on the total number of counts in the 20-80 keV band as follows. We require the signal-to-noise ratio $\mathrm{S} / \mathrm{N}=N_{\mathrm{S}} / \sqrt{N_{\mathrm{B}}+N_{\mathrm{S}}} \geq 5$, where $N_{\mathrm{S}}$ is the total number of source counts and $\sqrt{N_{\mathrm{B}}+N_{\mathrm{S}}}$ is the corresponding noise, with $N_{\mathrm{B}}=N_{\mathrm{ICM}}+N_{\mathrm{CXB}}+N_{\text {instr }}$ including all background counts (ICM, CXB and instrumental).

We approximate the instrumental background by a power law corresponding to $6 \times 10^{-3}$ counts $/ \mathrm{s} / \mathrm{keV} / \mathrm{FoV}$ at $10 \mathrm{keV}$ and $2 \times 10^{-4}$ counts $/ \mathrm{s} / \mathrm{keV} / \mathrm{FoV}$ at $50 \mathrm{keV}$, which are values adopted from the ASTRO-H Quick Reference (Table 2). For our purpose, a simple power-law approximation of the instrumental noise suffices, since uncertainties will be dominated by the modelling of the thermal and IC emission ${ }^{5}$. We convert the thermal bremsstrahlung and $\mathrm{CXB}$ fluxes into count rates by multiplying by the HXI effective area taken from the ASTRO-H Quick Reference (Fig. 1). Here, $N_{\mathrm{S}}$ is then related to the HXR flux density, $S_{\mathrm{x}}(E)=k_{\mathrm{x}} E^{-\alpha}$, through

$N_{\mathrm{S}}=T_{\mathrm{obs}} \int_{20 \mathrm{keV}}^{80 \mathrm{keV}} \frac{S_{\mathrm{x}}(E) A_{\mathrm{eff}}(E)}{E} \mathrm{~d} E$,

where $T_{\mathrm{obs}}$ is the observation time and $A_{\text {eff }}(E)$ the effective area. Using the $N_{\mathrm{S}}$ for which $S / N \geq 5$, we solve for $k_{\mathrm{x}}$ and, finally, calculate the corresponding flux as $F_{\mathrm{x}}=k_{\mathrm{x}} \int_{20 \mathrm{keV}}^{80 \mathrm{keV}} E^{-\alpha} \mathrm{d} E$. The spectral index used here is the same as above. This flux is then converted into a magnetic field, $B_{\mathrm{dt}}^{\mathrm{S} / \mathrm{N}}$, using

$$
\begin{aligned}
& \frac{F_{x} v_{r}^{-\alpha}}{s_{r} \int_{v_{\min }}^{v_{\max }} v_{x}^{-\alpha} \mathrm{d} v_{x}}= \\
& \frac{2.46 \times 10^{-19} T_{\mathrm{CMB}}^{3}}{B_{\mathrm{dt}}^{\mathrm{S} / \mathrm{N}}} \frac{b(p)}{a(p)}\left(\frac{4.96 \times 10^{3} T_{\mathrm{CMB}}}{B_{\mathrm{dt}}^{\mathrm{S} / \mathrm{N}}}\right)^{\alpha},
\end{aligned}
$$

where $T_{\mathrm{CMB}}=T_{0}(1+z)$ is the CMB temperature, $F_{\mathrm{x}}$ the X-ray flux, $s_{r}$ the radio flux density at $v_{\mathrm{r}}, p=2 \alpha+1$, and

$$
\begin{aligned}
& a(p)=\frac{2^{(p-1) / 2} \sqrt{3} \Gamma\left(\frac{3 p-1}{12}\right) \Gamma\left(\frac{3 p+19}{12}\right) \Gamma\left(\frac{p+7}{4}\right)}{16 \pi^{1 / 2} \Gamma\left(\frac{p+9}{4}\right)}, \\
& b(p)=2^{p+3} \frac{\left(p^{2}+4 p+11\right) \Gamma\left(\frac{p+5}{2}\right) \zeta\left(\frac{p+5}{2}\right)}{(p+3)^{2}(p+1)(p+5)} .
\end{aligned}
$$

Here $\Gamma$ and $\zeta$ are the gamma and Riemann zeta functions, respectively (Pacholczyk 1970; Blumenthal \& Gould 1970; Sarazin 1988; Longair 2011 $)^{6}$. In this approach, for objects whose size in the sky $\left(\Omega \operatorname{arcmin}^{2}\right)$ is larger than the HXI FoV $\left(9 \times 9 \operatorname{arcmin}^{2}\right)$, the radio flux density $s_{\mathrm{r}}$ is scaled down roughly as $9 \times 9 / \Omega$. This implies slightly conservative results since halos and relics are not uniform sources. Eventually, the resulting magnetic field value, $B_{\mathrm{dt}}^{\mathrm{S} / \mathrm{N}}$, is again a cap on the volume-average magnetic field below which HXR IC emission is detectable by ASTRO-H.

The analysis based on the signal-to-noise ratio is done assuming a straight power law in the $20-80 \mathrm{keV}$ energy band. That

\footnotetext{
5 Additionally, comparing our power-law estimate to the ASTRO-H response files (http://astro-h.isas.jaxa.jp/researchers/sim/ response.html) in the $20-80 \mathrm{keV}$ band shows that we overestimate the predicted instrumental background by just a few percent.

6 The arguments of the gamma function $(\Gamma)$ are slightly different with respect to the values when a coherent magnetic field is assumed.
} 
Table 4. Results for halos.

\begin{tabular}{|c|c|c|c|c|c|c|}
\hline Cluster & $\alpha$ & $\begin{array}{l}\text { Current limits } \\
\qquad B_{\mathrm{V}} \\
{[\mu \mathrm{G}]}\end{array}$ & $\begin{array}{c}\text { ASTRO-H } 1 \mathrm{Ms} \\
\qquad B_{\mathrm{dt}} \\
{[\mu \mathrm{G}]}\end{array}$ & $\begin{array}{c}B_{\mathrm{dt}}^{\mathrm{opt}} \\
{[\mu \mathrm{G}]}\end{array}$ & $\begin{array}{c}E \\
{[\mathrm{keV}]}\end{array}$ & $S_{x} \times E$ \\
\hline Bullet & 1.50 & 0.38 & 1.00 & 1.00 & 45 & 0.20 \\
\hline A0520 & 1.12 & & 0.37 & 0.57 & $33(16)$ & $0.14(0.06)$ \\
\hline A0521 & 1.81 & & 0.80 & 1.16 & $31(18)$ & $0.11(0.06)$ \\
\hline A0665 & 1.04 & 0.11 & 0.28 & 0.41 & $46(29)$ & $0.42(0.20)$ \\
\hline A0697 & 1.52 & & 0.33 & 0.33 & 53 & 0.29 \\
\hline A0754 & 1.10 & $0.09^{\gamma_{\max }=6.0 \times 10^{4}}$ & $0.45^{\gamma_{\max }=2.7 \times 10^{4}}$ & 0.45 & 52 & 0.47 \\
\hline A1300 & 1.76 & & 1.02 & 1.27 & $30(23)$ & $0.10(0.07)$ \\
\hline Coma & 1.16 & $0.32^{\gamma_{\max }=3.9 \times 10^{4}}$ & $0.36^{\gamma_{\max }=3.7 \times 10^{4}}$ & 0.36 & 48 & 3.67 \\
\hline A1758a & 1.50 & & 0.71 & 0.95 & 34 & 0.16 \\
\hline A1914 & 1.95 & $0.59_{\gamma_{\min }=2.3 \times 10^{3}}$ & $1.12_{\gamma_{\min }=1.7 \times 10^{3}}$ & 1.12 & 51 & 0.33 \\
\hline A 2163 & 1.18 & 0.13 & 0.49 & 0.49 & 57 & 0.82 \\
\hline A2219 & 0.72 & & 0.29 & 0.30 & $39(37)$ & $0.22(0.21)$ \\
\hline A 2255 & 1.60 & 0.32 & 0.60 & 0.77 & $46(34)$ & $0.42(0.27)$ \\
\hline A 2256 & 1.35 & 0.20 & 0.49 & 0.53 & 48 & 0.60 \\
\hline A2319 & 1.65 & 0.66 & 0.65 & 0.65 & 53 & 1.36 \\
\hline A 2744 & 0.92 & 0.29 & 0.44 & 0.59 & $39(26)$ & $0.21(0.12)$ \\
\hline A 3562 & 1.30 & $0.10^{\gamma_{\max }=6.8 \times 10^{4}}$ & $0.37^{\gamma_{\max }}=3.5 \times 10^{4}$ & 0.53 & $41(27)$ & $0.27(0.14)$ \\
\hline $\mathrm{CLO} 217+70^{a}$ & 1.20 & & 0.36 & 0.69 & $46(17)$ & $0.41(0.12)$ \\
\hline MACSJ0717.5+3745 & 1.23 & & 1.29 & 1.29 & 40 & 0.12 \\
\hline MACSJ1752.0+4440 & 1.60 & & 0.97 & 1.37 & $32(20)$ & $0.13(0.07)$ \\
\hline PLCK G171.9-40.7 ${ }^{a}$ & 1.84 & & 1.15 & 1.15 & 42 & 0.11 \\
\hline RXCJ1514.9-1523 ${ }^{a}$ & 1.60 & & 0.52 & 0.76 & $39(24)$ & $0.22(0.11)$ \\
\hline RXCJ2003.5-2323 ${ }^{a}$ & 1.32 & & 0.92 & 1.19 & $31(22)$ & $0.11(0.07)$ \\
\hline
\end{tabular}

Notes. For each object, we show $\alpha$ and report the values of the current lower limit on the volume-average magnetic field, $B_{\mathrm{V}}$, where available, and the detection sensitivity on the volume-average magnetic field, $B_{\mathrm{dt}}$, for which IC emission would be detectable by ASTRO-H in 1 Ms. Additionally, $B_{\mathrm{dt}}^{\mathrm{opt}}$ is an optimistic estimate of the latter that corresponds to the intersection of the sensitivity curve and the thermal ICM emission, thus assuming the CXB is fully known and perfectly subtracted. The last two columns show the energy where non-thermal X-rays start to dominate the background for $B_{\mathrm{dt}}\left(B_{\mathrm{dt}}^{\mathrm{opt}}\right)$, and the corresponding non-thermal flux density in units of in $10^{-12} \mathrm{erg} \mathrm{cm}^{-2} \mathrm{~s}^{-1}$, respectively. In the cases where $\gamma_{\mathrm{min}}$ and/or $\gamma_{\min }$ are calculated from the radio data and differ from the standard values that we adopt (see Sect. 3), we show them explicitly. ${ }^{(a)}$ Thermal ICM emission computed using the model by Zandanel et al. (2014) for the gas density.

is, we ignore any possible cutoff effect in that range, otherwise Eq. (7) would not be valid anymore. In most cases, the cutoffs are at much lower and higher energies. However, for A0085 and A1914 (see Fig. 3) and for A2063 and A0013 (see Appendix A for the corresponding figures), for example, this method could give slightly optimistic results.

In some cases (only radio relics, see Appendix A for the figures), the CXB dominates the thermal ICM emission, and the intersection point between the ASTRO-H sensitivity and the $\mathrm{CXB}$ emission is at an energy lower than the rise in the $\mathrm{CXB}$ spectrum. The corresponding results for $B_{\mathrm{dt}}$ should be taken with caution, and in fact, in these cases, the signal-to-noise approach gives more conservative results. We underline again that our simple approach is meant to be only a first approximation and that, in the following, we base all our conclusions only on the more robust signal-to-noise approach. Nevertheless, we note that the two approaches turn out to give similar results.

We eventually decided to exclude the A2218 halo and the $\mathrm{ZwCl0008.8-5215}$ western relic from the final modelling. This is due to their low radio flux and to the apparent presence of quite high $\gamma_{\min }$, or break in the radio spectra, that would render the IC search in these objects fairly impossible.

We show an example of synchrotron and IC modelling in Fig. 2 for the case of the radio halo hosted by A2255, and we present and discuss all results in the next sections.

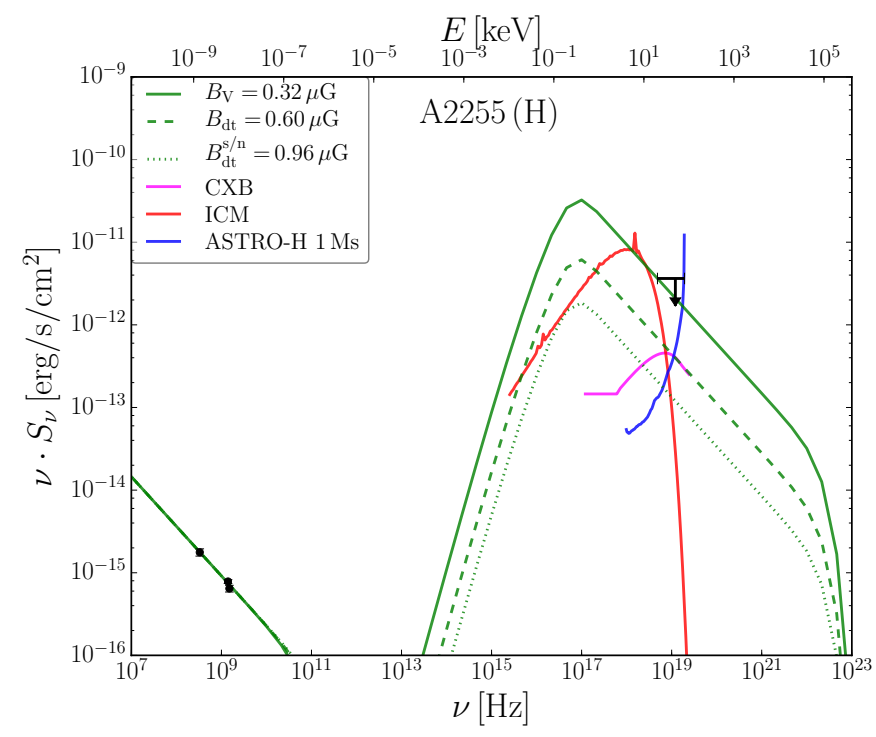

Fig. 2. Synchrotron and IC modelling of the radio halo (H) in A2255. The black points and arrow are the currently available radio data and HXR upper-limit, respectively, from Table 1. We show the thermal ICM and CXB emissions, together with the ASTRO-H HXI sensitivity for $1 \mathrm{Ms}$ of observations scaled to the halo angular size. The green lines show the models for the current volume-average lower limit $B_{\mathrm{V}}$ (solid), and for $B_{\mathrm{dt}}$ (dashed) and $B_{\mathrm{dt}}^{\mathrm{S} / \mathrm{N}}$ (dotted) for $1 \mathrm{Ms}$ of observations. 
Table 5. Results for relics.

\begin{tabular}{|c|c|c|c|c|c|c|c|}
\hline Cluster & Source & $\alpha$ & $\begin{array}{c}\text { Current limits } \\
\qquad B_{\mathrm{V}} \\
{[\mu \mathrm{G}]}\end{array}$ & $\begin{array}{c}\text { ASTRO-H 1Ms } \\
\qquad B_{\mathrm{dt}} \\
{[\mu \mathrm{G}]}\end{array}$ & $\begin{array}{c}B_{\mathrm{dt}}^{\mathrm{opt}} \\
{[\mu \mathrm{G}]}\end{array}$ & $\begin{array}{c}E \\
{[\mathrm{keV}]}\end{array}$ & $S_{x} \times E$ \\
\hline Toothbrush $^{a}$ & & 1.10 & & 1.65 & 2.13 & $31(22)$ & $0.11(0.07)$ \\
\hline A0013 & & 2.30 & & $1.98_{\gamma_{\min }=2.6 \times 10^{3}}$ & 1.98 & $31(31)$ & $0.06(0.06)$ \\
\hline A0085 & & 1.61 & $0.43^{\gamma_{\max }=1.6 \times 10^{4}}$ & $1.79^{\gamma_{\max }}=7.7 \times 10^{3}$ & 1.96 & $34(31)$ & $0.15(0.13)$ \\
\hline A0521 & & 1.45 & & 1.00 & 2.00 & $24(6)$ & $0.05(0.02)$ \\
\hline $\mathrm{A} 0610^{a}$ & & 1.40 & & 0.86 & 1.51 & $23(8)$ & $0.05(0.02)$ \\
\hline $\mathrm{AS} 753^{a}$ & & 2.10 & & $1.08^{\gamma_{\max }=2.0 \times 10^{4}}$ & 4.82 & $49(5)$ & $1.75(0.23)$ \\
\hline A0754 & & 1.10 & & 0.28 & 0.32 & $48(40)$ & $0.52(0.39)$ \\
\hline $\mathrm{A} 0781^{a}$ & & 1.23 & & 1.06 & 1.76 & $17(5)$ & $0.03(0.01)$ \\
\hline \multirow[t]{2}{*}{ A1240 } & $\mathrm{N}$ & 0.86 & & 0.16 & 0.28 & $28(5)$ & $0.07(0.02)$ \\
\hline & $\mathrm{S}$ & 0.71 & & 0.10 & 0.18 & $34(5)$ & $0.15(0.03)$ \\
\hline A1300 & & 0.90 & & 0.60 & 0.79 & 17 & 0.03 \\
\hline A1367 & & 1.90 & 0.89 & 0.76 & 2.16 & $43(10)$ & $0.29(0.05)$ \\
\hline $\mathrm{A} 1612^{a}$ & & 1.40 & & 1.64 & 3.39 & $23(5)$ & $0.05(0.02)$ \\
\hline Coma & & 1.18 & 0.87 & 0.28 & 0.65 & 49 (13) & $2.20(0.46)$ \\
\hline A1664 & & 1.10 & & 0.52 & 0.90 & 43 (18) & $0.29(0.10)$ \\
\hline $\mathrm{A} 2048^{a}$ & & 2.29 & & 2.43 & 2.96 & $20(15)$ & $0.04(0.03)$ \\
\hline A2061 & & 1.03 & & 0.31 & 0.48 & $34(16)$ & $0.15(0.06)$ \\
\hline A2063 & & 1.21 & $0.42^{\gamma_{\max }=1.4 \times 10^{4}}$ & $5.51^{\gamma_{\max }=4.0 \times 10^{3}}$ & 5.51 & 24 & 0.03 \\
\hline A 2163 & & 1.02 & & 0.75 & 0.99 & 16 & 0.03 \\
\hline A 2255 & & 1.40 & & 0.38 & 0.63 & 37 (17) & $0.19(0.08)$ \\
\hline A2256 & & 0.81 & & 0.34 & 0.41 & 49 (39) & $0.84(0.57)$ \\
\hline \multirow[t]{2}{*}{$\mathrm{A} 2345^{a}$} & $\mathrm{E}$ & 1.30 & & 0.49 & 0.99 & $40(14)$ & $0.22(0.06)$ \\
\hline & $\mathrm{W}$ & 1.56 & & 0.73 & 1.20 & 39 (19) & $0.20(0.09)$ \\
\hline $\mathrm{A} 2433^{a}$ & & 1.74 & & $1.38^{\gamma_{\max }}=9.3 \times 10^{3}$ & 2.52 & $27(5)$ & $0.07(0.02)$ \\
\hline A 2744 & & 1.10 & 0.35 & 0.51 & 1.09 & $31(6)$ & $0.11(0.03)$ \\
\hline \multirow[t]{2}{*}{ A3376 } & $\mathrm{E}$ & 0.88 & $0.13^{\gamma_{\max }=3.9 \times 10^{4}}$ & $0.30^{\gamma_{\max }=2.5 \times 10^{4}}$ & 0.59 & $49(16)$ & $1.78(0.45)$ \\
\hline & $\mathrm{W}$ & 1 & $0.26^{\gamma_{\max }=3.0 \times 10^{4}}$ & $0.48^{\gamma_{\max }=2.2 \times 10^{4}}$ & 1.32 & $49(5)$ & $0.67(0.09)$ \\
\hline $\mathrm{A} 3411^{a}$ & & 1 & & 0.38 & 0.59 & $45(25)$ & $0.34(0.14)$ \\
\hline \multirow[t]{2}{*}{ A3667 } & NW & 1.02 & 1.29 & 0.94 & 2.12 & 49 (12) & $1.37(0.27)$ \\
\hline & SE & 0.95 & & 0.31 & 0.53 & $49(21)$ & $0.96(0.32)$ \\
\hline A4038 & & 1.18 & $0.20^{\gamma_{\max }=2.5 \times 10^{4}}$ & $1.88^{\gamma_{\max }=8.2 \times 10^{3}}$ & 3.11 & $28(11)$ & $0.07(0.03)$ \\
\hline \multirow[t]{3}{*}{ El-Gordo $^{a}$} & $\mathrm{E}$ & 0.90 & & 0.12 & 0.12 & 51 & 0.10 \\
\hline & NW & 1.19 & & 0.77 & 0.77 & 51 & 0.10 \\
\hline & SE & 1.40 & & 0.41 & 0.41 & 51 & 0.10 \\
\hline Sausage $^{a}$ & & 1.06 & & $0.73^{\gamma_{\max }=5.4 \times 10^{4}}$ & 1.62 & $31(5)$ & $0.11(0.02)$ \\
\hline \multirow[t]{2}{*}{ MACSJ1149.5+2223 ${ }^{a}$} & $\mathrm{E}$ & 1.20 & & 1.63 & 1.63 & 5 & 0.01 \\
\hline & $\mathrm{W}$ & 0.80 & & 0.66 & 0.66 & 5 & 0.01 \\
\hline \multirow[t]{2}{*}{ MACSJ1752.0+4440 ${ }^{a}$} & $\mathrm{NE}$ & 1.16 & & 1.69 & 3.17 & $23(5)$ & $0.05(0.02)$ \\
\hline & SW & 1.10 & & 2.45 & 2.45 & 5 & 0.01 \\
\hline \multirow[t]{2}{*}{ PLCK G287.0+32.9 ${ }^{a}$} & $\mathrm{~N}$ & 1.26 & & 1.57 & 1.91 & $20(14)$ & $0.04(0.03)$ \\
\hline & $\mathrm{S}$ & 1.54 & & 2.26 & 4.14 & $17(5)$ & $0.03(0.01)$ \\
\hline \multirow[t]{2}{*}{ RXCJ1314.4-2515 } & $\mathrm{E}$ & 1.20 & & 0.62 & 1.18 & $23(5)$ & $0.05(0.02)$ \\
\hline & $\mathrm{W}$ & 1.40 & & 0.83 & 1.28 & $30(15)$ & $0.09(0.04)$ \\
\hline $\mathrm{ZwCl0008.8-5215^{a }}$ & $\mathrm{E}$ & 1.59 & & 0.85 & 2.14 & $39(9)$ & $0.21(0.04)$ \\
\hline \multirow[t]{2}{*}{$\mathrm{ZwCl} 2341.1+0000^{a}$} & $\mathrm{~N}$ & 0.49 & & 0.13 & 0.13 & 16 & 0.02 \\
\hline & $\mathrm{S}$ & 0.76 & & 0.19 & 0.29 & $32(16)$ & $0.12(0.05)$ \\
\hline
\end{tabular}

Notes. Columns are as in Table 4, with the addition of the second column which indicates the specific relic to which we are referring. ${ }^{(a)}$ Thermal ICM emission computed using the model by Zandanel et al. (2014) for the gas density. ${ }^{(b)}$ No improvement upon current constraints are possible according to this analysis (see main text for details). 
R. Bartels et al.: Inverse-Compton emission from clusters of galaxies
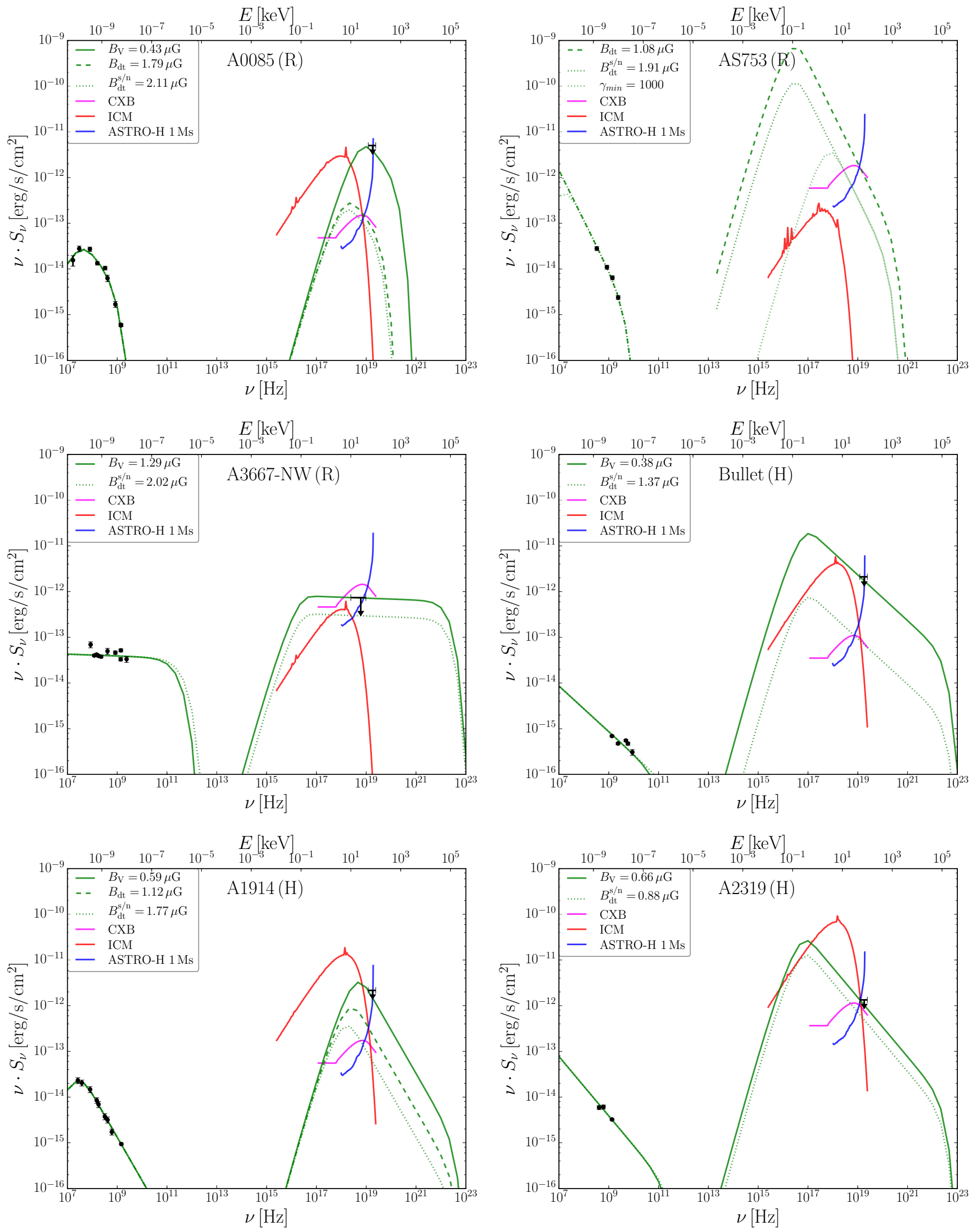

Fig. 3. Synchrotron and IC modelling of the A0085, AS753, and A3667-NW radio relics (R), and of the Bullet, A1914 and A2319 radio halos (H). Caption as in Fig. 2. For A3667-NW and A2319, the lines corresponding to $B_{\mathrm{dt}}$ are not shown as they correspond to that of $B_{\mathrm{V}}$. 
Table 6. Signal-to-noise results for halo.

\begin{tabular}{|c|c|c|c|c|c|c|c|c|}
\hline \multirow[b]{2}{*}{ Cluster } & \multirow[b]{2}{*}{$\begin{array}{c}s_{\mathrm{r}} \\
{[\mathrm{mJy}]}\end{array}$} & \multirow[b]{2}{*}{$\begin{array}{c}v_{\mathrm{r}} \\
{[\mathrm{MHz}]}\end{array}$} & \multicolumn{2}{|c|}{ ASTRO-H $100 \mathrm{ks}$} & \multicolumn{2}{|c|}{$500 \mathrm{ks}$} & \multicolumn{2}{|r|}{$1 \mathrm{Ms}$} \\
\hline & & & $\begin{array}{l}B_{\mathrm{dt}}^{\mathrm{S} / \mathrm{N}} \\
{[\mu \mathrm{G}]}\end{array}$ & $F_{20-80 \mathrm{keV}^{a}}$ & $\begin{array}{l}B_{\mathrm{dt}}^{\mathrm{S} / \mathrm{N}} \\
{[\mu \mathrm{G}]}\end{array}$ & $F_{20-80 \mathrm{keV}^{a}}$ & $\begin{array}{c}B_{\mathrm{dt}}^{\mathrm{S} / \mathrm{N}} \\
{[\mu \mathrm{G}]}\end{array}$ & $F_{20-80 \mathrm{keV}^{a}}$ \\
\hline Bullet & 52 & 1340 & 0.84 & 0.42 & 1.18 & 0.18 & 1.37 & 0.13 \\
\hline A 0520 & 85 & 325 & 0.26 & 0.40 & 0.39 & 0.17 & 0.46 & 0.12 \\
\hline A0521 & 328 & 153 & 0.61 & 0.28 & 0.83 & 0.12 & 0.94 & 0.08 \\
\hline A0665 & 197 & 327 & 0.25 & 0.72 & 0.38 & 0.31 & 0.45 & 0.22 \\
\hline A0697 & 135 & 153 & 0.25 & 0.85 & 0.35 & 0.37 & 0.40 & 0.26 \\
\hline A0754 & 86 & 1365 & 0.27 & 0.97 & 0.40 & 0.42 & 0.48 & 0.30 \\
\hline A1300 & 130 & 325 & 0.74 & 0.28 & 1.02 & 0.12 & 1.16 & 0.08 \\
\hline Coma* & 640 & 1400 & 0.20 & 1.88 & 0.29 & 0.83 & 0.34 & 0.59 \\
\hline$* *$ & " & " & 0.54 & $"$ & 0.79 & $"$ & 0.93 & $"$ \\
\hline$* * *$ & " & $"$ & 0.25 & 1.11 & 0.37 & 0.49 & 0.44 & 0.34 \\
\hline A1758 & 146 & 325 & 0.55 & 0.38 & 0.78 & 0.16 & 0.90 & 0.11 \\
\hline A1914 & 64 & 1465 & 1.18 & 0.53 & 1.57 & 0.23 & 1.77 & 0.16 \\
\hline $\mathrm{A} 2163^{*}$ & 861 & 325 & 0.46 & 1.08 & 0.67 & 0.47 & 0.79 & 0.33 \\
\hline$* *$ & " & 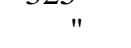 & 0.51 & $"$ & 0.75 & $"$ & 0.88 & $"$ \\
\hline A2219 & 232 & 325 & 0.19 & 0.66 & 0.31 & 0.28 & 0.38 & 0.20 \\
\hline A 2255 & 536 & 330 & 0.61 & 0.64 & 0.84 & 0.28 & 0.96 & 0.20 \\
\hline A2256* & 6600 & 63 & 0.41 & 0.87 & 0.58 & 0.38 & 0.68 & 0.27 \\
\hline$*$ & " & $"$ & 0.48 & " & 0.68 & $"$ & 0.79 & " \\
\hline A2319* & 1450 & 408 & 0.56 & 1.20 & 0.77 & 0.53 & 0.88 & 0.37 \\
\hline$* *$ & " & " & 0.79 & $"$ & 1.08 & $"$ & 1.23 & " \\
\hline A2744 & 218 & 325 & 0.32 & 0.55 & 0.49 & 0.23 & 0.59 & 0.16 \\
\hline A3562 & 20 & 1400 & 0.26 & 0.55 & 0.38 & 0.24 & 0.44 & 0.17 \\
\hline $\mathrm{CLO} 217+70^{a}$ & 326 & 325 & 0.33 & 0.68 & 0.48 & 0.29 & 0.56 & 0.21 \\
\hline MACSJ0717.5+3745 & 493 & 235 & 0.91 & 0.37 & 1.35 & 0.15 & 1.58 & 0.11 \\
\hline MACSJ1752.0+4440 & 164 & 323 & 0.73 & 0.34 & 1.02 & 0.14 & 1.17 & 0.10 \\
\hline PLCK G171.9-40.7 ${ }^{a}$ & 483 & 235 & 0.98 & 0.26 & 1.33 & 0.11 & 1.52 & 0.08 \\
\hline RXCJ1514.9-1523 ${ }^{a}$ & 102 & 327 & 0.46 & 0.44 & 0.63 & 0.19 & 0.73 & 0.13 \\
\hline RXCJ2003.5-2323 ${ }^{a}$ & 360 & 240 & 0.64 & 0.35 & 0.93 & 0.15 & 1.09 & 0.10 \\
\hline
\end{tabular}

Notes. For each halo, we show the radio data values $s_{\mathrm{r}}$ and $\nu_{\mathrm{r}}$ used in Eq. (7) to calculate $B_{\mathrm{dt}}^{\mathrm{S} / \mathrm{N}}$. We show the latter for $100 \mathrm{ks}, 500 \mathrm{ks}$ and, $1 \mathrm{Ms}$ of ASTRO-H observations, together with the corresponding flux in the $20-80 \mathrm{keV}$ energy band in units of $10^{-12} \mathrm{erg} \mathrm{cm}^{-2} \mathrm{~s}^{-1}$. Within a few percent error, the fluxes and magnetic fields scale with observation time according to Eq. (9). ${ }^{(*)}$ In this case, the source is larger than the HXI FoV of $9 \times 9=81 \operatorname{arcmin}^{2}$. In our work, for simplicity, we assumed a uniform surface brightness, therefore, $s_{r}$ was scaled by a factor $81 / x$ where $x$ arcmin ${ }^{2}$ is the size that a source covers in the sky (see Tables 1 and 2). This estimate results in slightly conservative predictions since halos and relics are non-uniform sources. ${ }^{(*)}$ This gives results that assume all radio emission is coming from an area equal to the HXI FoV, so $s_{\mathrm{r}}$ was not scaled. This can be considered an optimistic estimate for the detectable magnetic field. ${ }^{(* *)}$ Additional analysis for Coma. Since the Coma radio halo is extremely large in the sky, we estimated the thermal ICM emission only in the HXI FoV, integrating Eq. (4) out to $\sim 180 \mathrm{kpc}$, which roughly corresponds to the HXI FoV, rather than over the full halo size. ${ }^{(a)}$ Thermal ICM emission computed using the model by Zandanel et al. (2014) for the gas density.

\section{Results}

The results of our analysis are given in Tables 4 and 5 for radio halos and relics, respectively. The third column contains the current lower limit on the magnetic field for objects where an HXR flux upper limit is available in literature. Our lower limits on the magnetic field are slightly higher than those in the original works from which the HXR upper limits fluxes are taken because of our assumption on the magnetic field distribution (see Sect. 4). The sixth column contains $B_{\mathrm{dt}}$, which corresponds to the cap value of the volume-averaged magnetic field up to which ASTRO-H can still detect non-thermal IC for $1 \mathrm{Ms}$ of observation time. The eighth column shows an optimistic value for the detection sensitivity on the volume-average magnetic field, $B_{\mathrm{dt}}^{\mathrm{opt}}$, obtained assuming that the CXB can be perfectly subtracted and, therefore, always considering the intersection of the ASTRO-H sensitivity curve with the thermal ICM emission as estimation point for $B_{\mathrm{dt}}^{\mathrm{opt}}$ (see Sect. 7 for details).
In Tables 6 and 7, we show the estimate of the ASTRO-H detection sensitivity of the volume-average magnetic field, $B_{\mathrm{dt}}^{\mathrm{S} / \mathrm{N}}$, obtained with the signal-to-noise approach in the $20-80 \mathrm{keV}$ band, for halos and relics, respectively. We show the results for $100 \mathrm{ks}, 500 \mathrm{ks}$, and $1 \mathrm{Ms}$ of observation time.

Comparing the results in Tables $4-7$, it is clear that the results based on the intersection point between the background emission and the ASTRO-H sensitivity are more pessimistic. In particular, for sources larger than the HXI FoV (e.g., the halos of Coma and A2319, and the relics of Coma and A3667), our initial analysis shows little room for improvement on the current constraints, while the signal-to-noise analysis shows something different. The simplified assumption of a uniform source in our initial analysis is the most obvious reason for the pessimistic perspective for sources that cover a large area on the sky. This is also the origin for the $B_{\mathrm{dt}}$ values of the Coma and A2319 radio halos and the Coma and A3667-NW relic being at the same level of current upper limits. Additionally, assuming that the IC emission 
Table 7. Signal-to-noise results for relics.

\begin{tabular}{|c|c|c|c|c|c|c|c|c|c|}
\hline \multirow[b]{2}{*}{ Cluster } & \multirow[b]{2}{*}{ Source } & \multirow[b]{2}{*}{$\begin{array}{c}s_{\mathrm{r}} \\
{[\mathrm{mJy}]}\end{array}$} & \multirow[b]{2}{*}{$\begin{array}{c}v_{\mathrm{r}} \\
{[\mathrm{MHz}]}\end{array}$} & \multicolumn{2}{|c|}{ ASTRO-H $100 \mathrm{ks}$} & \multicolumn{2}{|c|}{$500 \mathrm{ks}$} & \multicolumn{2}{|r|}{$1 \mathrm{Ms}$} \\
\hline & & & & $\begin{array}{l}B_{\mathrm{dt}}^{\mathrm{S} / \mathrm{N}} \\
{[\mu \mathrm{G}]}\end{array}$ & $F_{20-80 \mathrm{keV}^{a}}$ & $\begin{array}{l}B_{\mathrm{dt}}^{\mathrm{S} / \mathrm{N}} \\
{[\mu \mathrm{G}]}\end{array}$ & $F_{20-80 \mathrm{keV}^{a}}$ & $\begin{array}{c}B_{\mathrm{dt}}^{\mathrm{S} / \mathrm{N}} \\
{[\mu \mathrm{G}]}\end{array}$ & $F_{20-80 \mathrm{keV}^{a}}$ \\
\hline Toothbrush $^{a}$ & & 7600 & 74 & 1.08 & 0.35 & 1.64 & 0.15 & 1.95 & 0.10 \\
\hline A0013 & & 6000 & 80 & 1.10 & 0.14 & 1.45 & 0.06 & 1.63 & 0.04 \\
\hline A0085 & & 8330 & 160 & 1.33 & 0.37 & 1.84 & 0.16 & 2.11 & 0.11 \\
\hline A0521 & & 297 & 153 & 0.54 & 0.23 & 0.78 & 0.09 & 0.91 & 0.06 \\
\hline $\mathrm{A} 0610^{a}$ & & 59 & 600 & 0.47 & 0.22 & 0.69 & 0.09 & 0.80 & 0.06 \\
\hline $\operatorname{AS} 753 * a$ & & 460 & 1398 & 1.30 & 0.48 & 1.70 & 0.21 & 1.91 & 0.15 \\
\hline$* *$ & & $"$ & " & 2.02 & $"$ & 2.65 & $"$ & 2.97 & " \\
\hline A0754* & & 1450 & 74 & 0.22 & 0.80 & 0.33 & 0.35 & 0.39 & 0.24 \\
\hline$* *$ & & & & 0.25 & " & 0.37 & " & 0.44 & $"$ \\
\hline $\mathrm{A} 0781^{a}$ & & 93 & 325 & 0.48 & 0.21 & 0.72 & 0.09 & 0.85 & 0.06 \\
\hline \multirow[t]{2}{*}{ A1240 } & $\mathrm{N}$ & 21 & 325 & 0.09 & 0.32 & 0.14 & 0.13 & 0.17 & 0.09 \\
\hline & $\mathrm{S}$ & 29 & 325 & 0.06 & 0.46 & 0.10 & 0.20 & 0.12 & 0.14 \\
\hline A1300 & & 75 & 325 & 0.27 & 0.24 & 0.44 & 0.10 & 0.54 & 0.07 \\
\hline A1367 & & 180 & 610 & 0.78 & 0.43 & 1.04 & 0.19 & 1.18 & 0.13 \\
\hline $\mathrm{A} 1612^{a}$ & & 776 & 241 & 0.92 & 0.23 & 1.34 & 0.09 & 1.56 & 0.06 \\
\hline Coma* & & 3300 & 151 & 0.27 & 0.70 & 0.40 & 0.30 & 0.47 & 0.21 \\
\hline$* *$ & & " & " & 0.56 & $"$ & 0.82 & $"$ & 0.97 & $"$ \\
\hline A1664 & & 1250 & 150 & 0.45 & 0.58 & 0.67 & 0.25 & 0.79 & 0.17 \\
\hline $\mathrm{A} 2048^{a}$ & & 559 & 325 & 1.41 & 0.15 & 1.86 & 0.06 & 2.08 & 0.04 \\
\hline A2061 & & 104 & 327 & 0.20 & 0.42 & 0.31 & 0.18 & 0.36 & 0.12 \\
\hline A2063 & & 13200 & 178 & 2.25 & 0.17 & 3.46 & 0.07 & 4.12 & 0.05 \\
\hline A2163 & & 82 & 325 & 0.30 & 0.22 & 0.48 & 0.09 & 0.57 & 0.06 \\
\hline A2255 & & 103 & 330 & 0.22 & 0.42 & 0.31 & 0.18 & 0.36 & 0.13 \\
\hline $\mathrm{A} 2256^{* a}$ & & 5600 & 63 & 0.26 & 0.94 & 0.41 & 0.41 & 0.50 & 0.29 \\
\hline$* *$ & & & " & 0.37 & $"$ & 0.58 & " & 0.71 & \\
\hline \multirow[t]{2}{*}{$\mathrm{A} 2345^{a}$} & $\mathrm{E}$ & 188 & 325 & 0.41 & 0.47 & 0.59 & 0.20 & 0.68 & 0.14 \\
\hline & $\mathrm{W}$ & 291 & 325 & 0.63 & 0.42 & 0.88 & 0.18 & 1.02 & 0.12 \\
\hline $\mathrm{A} 2433^{a}$ & & 5310 & 74 & 0.90 & 0.23 & 1.24 & 0.10 & 1.42 & 0.07 \\
\hline $\mathrm{A} 2744$ & & 98 & 325 & 0.33 & 0.37 & 0.50 & 0.15 & 0.59 & 0.11 \\
\hline A3376* & $\mathrm{E}$ & 122 & 1400 & 0.11 & 0.78 & 0.17 & 0.34 & 0.21 & 0.24 \\
\hline$* *$ & & & $"$ & 0.23 & $"$ & 0.36 & " & 0.43 & $"$ \\
\hline$*$ & W & 113 & 1400 & 0.24 & 0.74 & 0.36 & 0.32 & 0.43 & 0.23 \\
\hline$* *$ & & & " & 0.29 & & 0.44 & & 0.53 & $"$ \\
\hline $\mathrm{A} 3411^{a}$ & & 2074 & 74 & 0.38 & 0.66 & 0.58 & 0.29 & 0.69 & 0.20 \\
\hline A $3667 *$ & NW & 81000 & 86 & 1.12 & 0.74 & 1.70 & 0.32 & 2.02 & 0.23 \\
\hline$* *$ & & & $"$ & 1.95 & & 2.94 & & 3.51 & \\
\hline$*$ & SE & 3200 & 120 & 0.30 & 0.77 & 0.46 & 0.33 & 0.55 & 0.23 \\
\hline$* *$ & & & $"$ & 0.44 & " & 0.68 & $"$ & 0.81 & $"$ \\
\hline A4038 & & 380 & 606 & 0.67 & 0.29 & 1.00 & 0.12 & 1.18 & 0.08 \\
\hline Sausage $^{a}$ & & 96 & 1400 & 0.55 & 0.36 & 0.84 & 0.15 & 1.00 & 0.11 \\
\hline \multirow[t]{3}{*}{ El-Gordo $^{a}$} & E & 1 & 610 & 0.06 & 0.44 & 0.10 & 0.19 & 0.12 & 0.13 \\
\hline & NW & 19 & 610 & 0.47 & 0.40 & 0.69 & 0.17 & 0.82 & 0.12 \\
\hline & SE & 3 & 610 & 0.30 & 0.37 & 0.42 & 0.16 & 0.49 & 0.11 \\
\hline \multirow[t]{2}{*}{ MACSJ1149.5+2223 ${ }^{a}$} & $\mathrm{E}$ & 23 & 323 & 0.40 & 0.14 & 0.63 & 0.05 & 0.75 & 0.04 \\
\hline & $\mathrm{W}$ & 17 & 323 & 0.18 & 0.17 & 0.31 & 0.06 & 0.38 & 0.04 \\
\hline \multirow[t]{2}{*}{ MACSJ1752.0+4440 ${ }^{a}$} & $\mathrm{NE}$ & 410 & 323 & 0.90 & 0.25 & 1.36 & 0.10 & 1.61 & 0.07 \\
\hline & SW & 163 & 323 & 0.66 & 0.17 & 1.03 & 0.07 & 1.24 & 0.05 \\
\hline \multirow[t]{2}{*}{ PLCK G287.0+32.9 $9^{a}$} & $\mathrm{~N}$ & 550 & 150 & 0.77 & 0.23 & 1.14 & 0.09 & 1.35 & 0.06 \\
\hline & $\mathrm{S}$ & 780 & 150 & 1.05 & 0.19 & 1.51 & 0.08 & 1.75 & 0.05 \\
\hline \multirow[t]{2}{*}{ RXCJ1314.4-2515 } & $\mathrm{E}$ & 28 & 610 & 0.33 & 0.24 & 0.49 & 0.10 & 0.58 & 0.07 \\
\hline & $\mathrm{W}$ & 65 & 610 & 0.55 & 0.30 & 0.80 & 0.12 & 0.93 & 0.09 \\
\hline ZwCl0008.8-5215 & $\mathrm{E}$ & 820 & 241 & 0.72 & 0.41 & 1.00 & 0.18 & 1.15 & 0.12 \\
\hline \multirow{2}{*}{$\mathrm{ZwCl} 2341.1+0000^{a}$} & $\mathrm{~N}$ & 36 & 157 & 0.05 & 0.24 & 0.10 & 0.09 & 0.13 & 0.06 \\
\hline & $\mathrm{S}$ & 70 & 157 & 0.10 & 0.42 & 0.17 & 0.18 & 0.21 & 0.12 \\
\hline
\end{tabular}

Notes. Columns, and footnotes, are as in Table 6 , with the addition of the second column, which indicates the specific relic to which we are referring.

is only detectable at energies where it dominates the background is a conservative approach, given that backgrounds are known and can be modelled in detail in a real analysis. Indeed, as already stressed earlier, this simple method is meant to be just a first-order approximation, and we take our signal-to-noise approach as reference when drawing conclusions.
The soft X-ray (SXR) and extreme ultra-violet (EUV) bands could also be promising for the detection of IC emission, since the flux would be much higher at those lower frequencies (e.g., Sarazin \& Lieu 1998). However, this is only advantageous if the background can be well constrained, and it crucially depends on the low-energy cutoff of the relativistic electron population, $\gamma_{\min }$, 
which is, in most cases, unconstrained by current radio data. Indeed, assuming an electron spectrum cutoff of $\gamma_{\min } \sim 10^{2}$, as discussed in Sect. 3, implies IC spectra peaking in the EUV band and often dominating the X-ray background in SXRs, in particular in case of very steep radio spectra. However, such steep spectra down to very low frequencies seem unlikely. The radio spectrum depends on the acceleration history of relativistic electrons, and as an example, steady state injection models feature a spectral break and would imply a low-frequency flattening (e.g., Sarazin 1999). In the near future, low-frequency observations with LOFAR (Röttgering et al. 2012) will provide crucial information and certainly shed more light on the spectrum of cosmicray electrons in clusters. This will be vital for assessing the possible IC contribution in the SXR and EUV regimes.

\subsection{Promising targets}

To pinpoint the most promising targets for ASTRO-H HXR observations, we focus on magnetic field estimates (e.g., Govoni \& Feretti 2004). FR measurements range from $\sim \mu \mathrm{G}$ for merging clusters up to $10 \mu \mathrm{G}$ for cool-core clusters (Carilli \& Taylor 2002; Clarke 2004; Vogt \& Enßlin 2005; Bonafede et al. 2010, 2013). Therefore, $\mu \mathrm{G}$-level magnetic fields in clusters are nowadays widely acknowledged.

Analytical estimates can be obtained by the equipartition argument, that is, by minimising the total energy content (the sum of the energy in the magnetic field and that in relativistic particles) of a radio source. While standard equipartition estimates, $B_{\text {eq }}$, are based on computing the synchrotron radio luminosity between two fixed frequencies, the so-called revised equipartition estimates, $B_{\mathrm{eq}}^{\text {rev }}$, are based on integrating over the relativistic electron distribution (Brunetti et al. 1997; Beck \& Krause 2005). In Table 8, we summarise all the (revised) equipartition estimates that we could find in literature for the clusters in our sample.

We stress that the equipartition estimates compiled in Table 8 should only be considered as reference values for comparison purposes. Not only do these suffer from several uncertainties, such as the ratio of electrons to protons in the source and the low-energy cutoff of the electron distribution, which are, a priori, unknown (see Govoni \& Feretti 2004 for a discussion), but also one could question whether the equipartition assumption is justified because the evolution of relativistic electrons and magnetic fields in clusters may be decoupled given the short lifetime of the electrons compared to that of clusters. We also note that different authors often adopt different assumptions when applying the equipartition argument.

We consider a given halo or relic to be a promising candidate for ASTRO-H if its $B_{\mathrm{dt}}^{\mathrm{S} / \mathrm{N}}$ in $100 \mathrm{ks}$ of observations is $\geq 1 \mu \mathrm{G}$; while if smaller, we consider it promising when that value is comparable to, or higher than, the corresponding (revised) equipartition estimate (where available). The value of $1 \mu \mathrm{G}$ is just a rough educated guess that assumes the magnetic field value of the Coma cluster as prototype for which the volume average is $\sim 2 \mu \mathrm{G}$ (Bonafede et al. 2010, 2013). We summarise all objects that meet these criteria in Table 9.

There is a crucial point to be considered when looking at our summary list of promising targets in Table 9, the size in the sky of a given halo. Halo size plays a crucial role in our predictions. Several objects in Table 9 have a size well below the ASTRO-H HXI FoV of $9 \times 9=81 \mathrm{arcmin}^{2}$. The smaller the size of a given object with respect to the FoV, the higher the contamination given by the thermal ICM emission, which obviously peaks in the centre of clusters. This is particularly important for
Table 8. Magnetic field equipartition estimates.

\begin{tabular}{|c|c|c|c|c|}
\hline Cluster & & $B_{\text {eq }}(\mu \mathrm{G})$ & $B_{\mathrm{eq}}^{\mathrm{rev}}(\mu \mathrm{G})$ & Reference \\
\hline \multicolumn{5}{|c|}{ Halos } \\
\hline Bullet & & & 1.2 & 1 \\
\hline A0520 & & 1.43 & & 2 \\
\hline A0665 & & 0.61 & & 3 \\
\hline A0754 & & 0.66 & & 4 \\
\hline Coma & & 0.45 & $0.7-1.9$ & $1,5,6,21$ \\
\hline A1914 & & 0.55 & 1.3 & 1,4 \\
\hline A2163 & & 0.70 & 0.97 & 1,3 \\
\hline A2219 & & 0.4 & 0.7 & 7 \\
\hline A2255 & & 0.53 & & 8 \\
\hline A 2256 & & $>1$ & 1.1 & 1,9 \\
\hline A2319 & & 0.53 & & 10 \\
\hline A2744 & & 0.5 & 1.0 & 1,7 \\
\hline A3562 & & 0.4 & & 11 \\
\hline MACSJ0717.5+3745 & & 3.43 & 6.49 & 12 \\
\hline RXCJ2003.5-2323 & & & 1.7 & 13 \\
\hline \multicolumn{5}{|c|}{ Relics } \\
\hline Toothbrush & & & $7.4-9.2$ & 14 \\
\hline A0085 & & $1.1-2.4$ & & 15,20 \\
\hline A0754 & & 0.3 & & 15 \\
\hline AS753 & & 1.3 & & 15 \\
\hline \multirow[t]{2}{*}{ A 1240} & $\mathrm{~N}$ & 1 & 2.4 & 16 \\
\hline & $\mathrm{S}$ & 1 & 2.5 & 16 \\
\hline A1367 & & 1 & & 15 \\
\hline Coma & & 0.55 & $0.7-1.7$ & $5,6,21$ \\
\hline A1664 & & 0.88 & & 2,17 \\
\hline A 2163 & & 2.2 & & 22 \\
\hline A2255 & & 0.53 & & 8 \\
\hline A2256 & & $>1$ & & 9 \\
\hline \multirow[t]{2}{*}{ A 2345} & $\mathrm{E}$ & 0.8 & 2.2 & 16 \\
\hline & $\mathrm{W}$ & 1 & 2.9 & 16 \\
\hline A2744 & & 0.6 & 1.3 & 7 \\
\hline A3667 & NW & $1.5-2.5$ & & 6,18 \\
\hline A 4038 & & 3 & & 17 \\
\hline ZwCl0008.8-5215 & $\mathrm{E}$ & 2.5 & 6.6 & 19 \\
\hline \multirow{2}{*}{$\mathrm{ZwCl} 2341.1+0000$} & $\mathrm{~N}$ & 0.59 & 0.64 & 23 \\
\hline & $\mathrm{E}$ & 0.54 & 0.66 & 23 \\
\hline
\end{tabular}

Notes. Magnetic field estimates based on the equipartition theorem. $B_{\mathrm{eq}}$ is the equipartition magnetic field, while $B_{\mathrm{eq}}^{\text {rev }}$ is the so-called revised equipartition magnetic field.

References. (1) Petrosian et al. (2006); (2) Govoni et al. (2001); (3) Feretti et al. (2004); (4) Bacchi et al. (2003); (5) Giovannini et al. (1991); (6) Govoni \& Feretti (2004); (7) Orru et al. (2007); (8) Feretti et al. (1997a); (9) Clarke \& Ensslin (2006); (10) Feretti et al. (1997b); (11) Venturi et al. (2003); (12) Pandey-Pommier et al. (2013); (13) Giacintucci et al. (2009); (14) van Weeren et al. (2012c); (15) Chen et al. (2008); (16) Bonafede et al. (2009b); (17) Kale \& Dwarakanath (2012); (18) Johnston-Hollitt (2004); (19) van Weeren et al. (2011b); (20) Ensslin et al. (1998); (21) Thierbach et al. (2003); (22) Feretti et al. (2001).

radio relics in the cluster's outskirts, which are the best targets for HXR IC detection, as is clear from the results presented in the tables in this work. On the other hand, an object that is much larger than the ASTRO-H FoV will call for multiple pointings with the corresponding increase in observation time. Regarding their morphology, ASTRO-H observation simulations of these objects are needed in order to give a definitive answer to which the most promising targets are. 
Table 9. Promising targets for ASTRO-H observations.

\begin{tabular}{|c|c|c|c|c|c|c|c|c|c|c|}
\hline Cluster & Source & Type & $z$ & Size & $\alpha$ & $B_{\mathrm{dt}}^{\mathrm{S} / \mathrm{N}}(100 \mathrm{ks})$ & $B_{\mathrm{dt}}^{\mathrm{S} / \mathrm{N}}(1 \mathrm{Ms})$ & $B_{\mathrm{dt}}(1 \mathrm{Ms})$ & $B_{\text {eq }}$ & $B_{\text {eq }}^{\text {rev }}$ \\
\hline Bullet & & $\mathrm{H}$ & 0.30 & 19 & 1.5 & 0.84 & 1.37 & 1.00 & & 1.2 \\
\hline Toothbrush $^{a}$ & & $\mathrm{R}$ & 0.23 & 18 & 1.1 & 1.08 & 1.95 & 1.65 & & $7.4-9.2$ \\
\hline $\mathrm{A}_{00013^{a}}$ & & $\mathrm{R}$ & 0.09 & 5 & 2.3 & 1.10 & 1.63 & 1.98 & & \\
\hline A0085 & & $\mathrm{R}$ & 0.06 & 26 & 1.6 & 1.33 & 2.11 & 1.79 & $1.1-2.4$ & \\
\hline $\mathrm{AS} 753^{a}$ & & $\mathrm{R}$ & 0.01 & 318 & 2.1 & 1.30 & 1.91 & 1.08 & 1.3 & \\
\hline \multirow[t]{2}{*}{ Coma } & & $\mathrm{H}$ & 0.02 & 710 & 1.2 & 0.20 & 0.34 & 0.36 & 0.5 & $0.7-1.9$ \\
\hline & & $\mathrm{R}$ & & 400 & 1.2 & 0.27 & 0.47 & 0.28 & 0.6 & $0.7-1.7$ \\
\hline A1914 & & $\mathrm{H}$ & 0.17 & 30 & 1.9 & 1.18 & 1.77 & 1.12 & 0.6 & 1.3 \\
\hline A $2048^{a}$ & & $\mathrm{R}$ & 0.10 & 7 & 2.3 & 1.41 & 2.08 & 2.43 & & \\
\hline A2063 & & $\mathrm{R}$ & 0.03 & 3 & 1.2 & 2.25 & 4.12 & 5.51 & & \\
\hline A2255 & & $\mathrm{H}$ & 0.08 & 79 & 1.6 & 0.61 & 0.96 & 0.60 & 0.5 & \\
\hline A2319 & & $\mathrm{H}$ & 0.06 & 198 & 1.7 & 0.56 & 0.88 & 0.65 & 0.5 & \\
\hline A3667 & NW & $\mathrm{R}$ & 0.06 & 250 & 1.0 & 1.12 & 2.02 & 0.94 & $1.5-2.5$ & \\
\hline \multirow{2}{*}{ PLCK G287.0+32.9 ${ }^{a}$} & $\mathrm{~N}$ & $\mathrm{R}$ & 0.39 & 7 & 1.3 & 0.77 & 1.35 & 1.57 & & \\
\hline & $\mathrm{S}$ & $\mathrm{R}$ & 0.39 & 6 & 1.5 & 1.05 & 1.75 & 4.14 & & \\
\hline
\end{tabular}

Notes. We summarise all objects that meet the criteria for being considered promising targets (see main text for details) and the corresponding magnetic field values testable by ASTRO-H in $100 \mathrm{ks}$ and $1 \mathrm{Ms}$ of observations in $\mu \mathrm{G}$. We show the most optimistic values obtained adopting the signal-to-noise approach, $B_{\mathrm{dt}}^{\mathrm{S} / \mathrm{N}}$, and also the more pessimistic estimates based on the intersection point between the background emission and the ASTRO-H sensitivity, $B_{\mathrm{dt}}$, for $1 \mathrm{Ms}$ of observations. We indicate the type of source, either radio halo $(\mathrm{H})$ or radio relic $(\mathrm{R})$, and, in case more than one relic is present in a given cluster, we explicitly mention which source we are referring to. We also report the approximate surface on the sky in $\operatorname{arcmin}^{2}$ and the spectral index $\alpha$. Finally, we also show (revised) equipartition estimates, in $\mu \mathrm{G}$, where available. ${ }^{(a)}$ Thermal ICM emission computed using the model by Zandanel et al. (2014) for the gas density.

We included Coma in our list of promising targets mainly because it is one of the best-studied clusters, and its observation by ASTRO-H is desirable. In fact, Coma represents one of the best targets for detecting and mapping the bulk of the ICM turbulent velocities for the first time with the Soft X-ray Spectrometer (SXS) on-board ASTRO-H (Kitayama et al. 2014). However, it does not represent the best hopes for the HXR IC detection.

To facilitate interpretation of our results, we provide a simple, approximate scaling of the ASTRO-H observation time with the magnetic field value $B_{\mathrm{dt}}^{\mathrm{S} / \mathrm{N}}$ that corresponds to the signal-tonoise approach. We note that the number of background counts scales linearly with time $N_{\mathrm{B}} \propto T_{\mathrm{obs}}$, and under the assumption that $N_{\mathrm{B}} \gg N_{\mathrm{S}}$, one finds that the number of source counts needed to reach a fixed signal-to-noise ratio scales as $N_{\mathrm{S}} \sim \sqrt{T_{\text {obs }}}$. From Eq. (6), $F_{x} \propto N_{\mathrm{S}} / T_{\mathrm{obs}} \propto T_{\mathrm{obs}}^{-1 / 2}$, and eventually, from Eq. (7), we find that, for any given $\alpha$,

$B_{\mathrm{dt}}^{\mathrm{S} / \mathrm{N}} \propto T_{\mathrm{obs}}^{\frac{1}{2(1+\alpha)}}$

Analogously, it can be shown that for a fixed observation time, i.e., fixed $N_{\mathrm{B}}$, the detectable magnetic field depends on the signal-to-noise ratio as follows:

$B_{\mathrm{dt}}^{\mathrm{S} / \mathrm{N}} \propto(\mathrm{S} / \mathrm{N})^{-\frac{1}{(1+\alpha)}}$.

\subsection{Comments on individual clusters}

We report in the following some comments on the individual clusters in our final list of the most promising objects - A0085, AS753, A3667, Bullet, A1914, A2255, A2319, and Coma while we leave the rest for Appendix A. We show the corresponding synchrotron and IC modelling in Figs. 2-4.

A0085. As can be seen from the top left-hand panel of Fig. 3, the radio relic of A0085 appears to have a low-energy cutoff in the radio spectrum. While this cutoff does not seem to affect our conclusion for the ASTRO-H detectability, if the magnetic field is much larger than $B_{\mathrm{S} / \mathrm{N}}$, the low-energy turnover of the spectrum would move to higher energies and could affect the detectability estimates. The current HXR upper limit for this cluster (Ajello et al. 2010) refers to a much larger area than that of the relic. We remind the reader that the result of the signal-tonoise approach should be taken with caution in this case because its assumes a straight power law in the $20-80 \mathrm{keV}$ range, while some spectral features could be expected.

AS753. The top right-hand panel of Fig. 3 shows the synchrotron and IC modelling for the radio relic hosted by this cluster. There are two things that should be noted: first, the very steep radio spectrum that implies a very-large IC flux in SXRs if we stick to the theoretically motivated $\gamma_{\min }=200$. For this reason, we also show in the figure the case with $\gamma_{\min }=1000$ and see that it does not change our predictions much for the HXR regime. Second, we must note that, since we lacked information on the gas density in this cluster, we used the phenomenological ICM model of Zandanel et al. (2014). This is, obviously, a source of a uncertainty and has very likely resulted in an underestimation of the gas density at the relic location.

A3667. This cluster hosts the prototype double relic system (Rottgering et al. 1997). The $1.9 \mathrm{Mpc}$ northwestern relic contains most of the diffuse emission. HXR emission from the A3667-NW relic has been studied by Ajello et al. (2009) and Nakazawa et al. (2009). We used the latter group's upper limit on the HXR non-thermal emission since it specifically refers to the relic region. In addition, a detailed X-ray study of the ICM can be found in Finoguenov et al. (2010) and Akamatsu et al. (2012). We show the corresponding synchrotron and IC modelling in the central left panel of Fig. 3. The current HXR upper limit is at the same level of our $B_{\mathrm{dt}}$ prediction due to our assumption of uniform radio sources. We try to assess this issue in Table 7 by providing an alternative estimate by assuming that all the radio emission comes from a region within the ASTRO-H HXI FoV. 

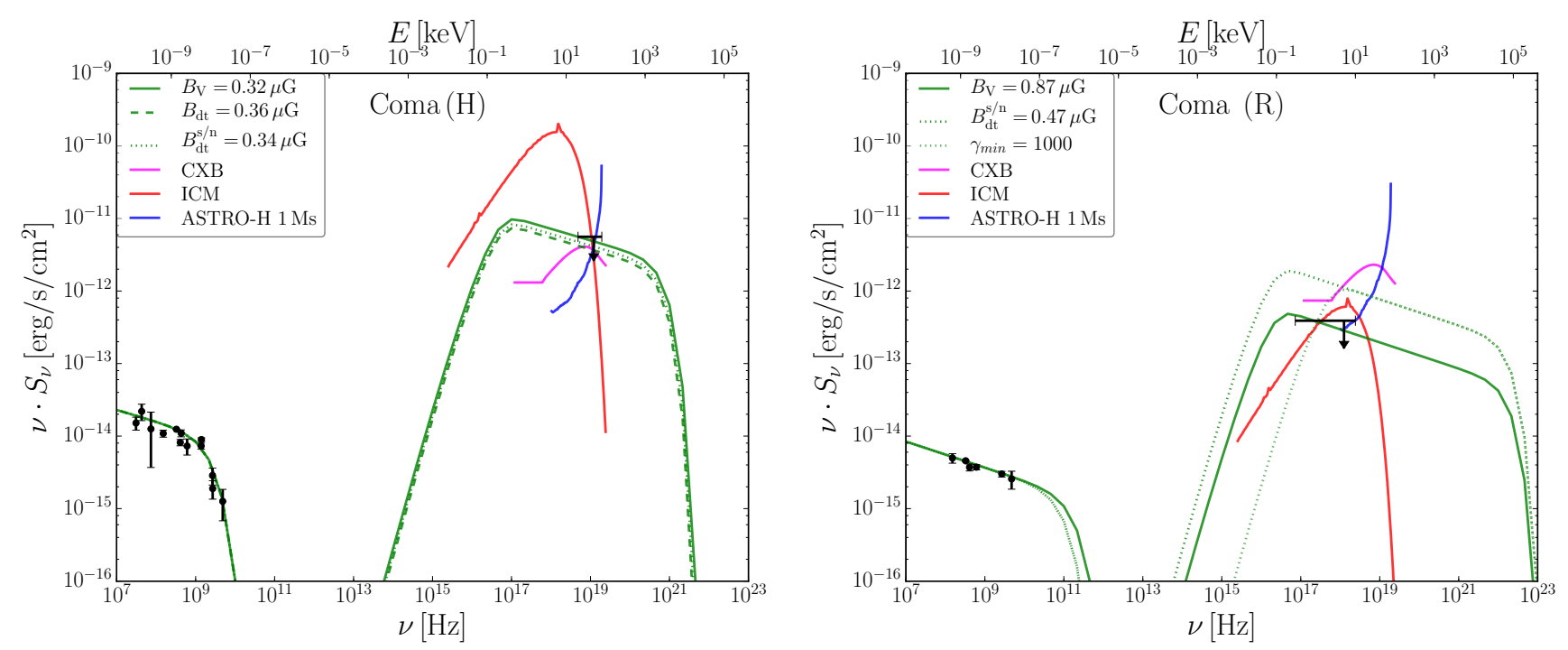

Fig. 4. Synchrotron and IC modelling of the radio halo $(\mathrm{H})$ and relic $(\mathrm{R})$ hosted by Coma. Caption as in Fig. 2. In the case of the relic, we do not show the line corresponding to $B_{\mathrm{dt}}$ because it gives worst estimates than that of $B_{\mathrm{V}}$. This is due to our assumption of uniform radio sources which is particularly inappropriate in case of objects with a large size in the sky, like Coma. See main text for details.

Recently, Kitayama et al. (2014) have pointed out that the A3667-NW relic is one of the more promising targets for ASTRO-H to detect non-thermal HXR emission. They predict that HXI can constrain the magnetic field to $B \geq 4 \mu \mathrm{G}$ in $200 \mathrm{ks}$ of observations. This is significantly higher than our predicted values of Tables 5 and 7 by about a factor of two. However, their approach is quite different because they estimate the $90 \%$ confidence-level (CL) upper limit obtainable with ASTRO-H in $200 \mathrm{ks}$ of observations. Moreover, the discrepancy mostly occurs because they assume that the SXR observed by Finoguenov et al. (2010) with XMM-Newton has a non-thermal origin, a hypothesis that cannot be excluded, and extrapolate this to HXRs with a power-law spectrum with a photon index of $\alpha=2$.

Bullet. We included the Bullet cluster for the broad interest in it and because it has been recently observed by NuSTAR, even if it does not fulfil our criteria of being in the promising targets list. Wik et al. (2014) have recently reported the results of the NuSTAR observations of the Bullet cluster where they do not find any evidence of non-thermal HXR emission from the radio halo. We derive slightly better prospects for ASTRO-H than their derived upper limit, as can be seen in the central right panel of Fig. 3. Although ASTRO-H and NuSTAR show comparable performances in HXR, the former has the advantage of the simultaneous operation of the SXI, allowing for a better determination of the thermal ICM emission.

A1914. The radio halo of A1914 has a very steep spectrum (Bacchi et al. 2003; Komissarov \& Gubanov 1994). While we modelled the low-energy flattening in radio with a spectral cutoff, the data is insufficient to distinguish it from a broken power law, as can be seen in the bottom left-hand panel of Fig. 3. In this sense, therefore, the result of the signal-to-noise approach should be taken with caution because its assumes a straight power law in the 20-80 keV range, while some spectral features could be expected. The X-ray upper limit is from Ajello et al. (2010).

A2255. This cluster hosts both a radio halo and a relic (Feretti et al. 1997a). Since the relic is at $\sim 10^{\prime}$ of the cluster centre and the XMM-Newton FoV is $30^{\prime}$, current HXR upper limits likely contain contributions from both the halo and the relic (Turner et al. 2001; Wik et al. 2012). The synchrotron and IC emission modelling of the radio halo is shown in Fig. 2. While we use radio observations from Feretti et al. (1997a) and Govoni et al. (2005), more recent observations were performed by Pizzo \& de Bruyn (2009). However, considering the different areas used for the flux extraction, the later results are comparable to the former for the purpose of our modelling.

A2319. We used the HXR upper limit from Ajello et al. (2009) and point out that we obtain a more constraining lower limit $B_{\mathrm{V}}>0.7 \mu \mathrm{G}$ as a result of our assumptions regarding the magnetic field distribution (see Sect. 4). The bottom right-hand panel of Fig. 3 shows the corresponding synchrotron and IC modelling. The current HXR upper limit is at the same level of our $B_{\mathrm{dt}}$ prediction owing to our assumption of uniform radio sources, and we try to assess this issue in Table 6 by providing an alternative estimate by assuming that all the radio emission comes from a region within the ASTRO-H HXI FoV.

Coma. We included the Coma cluster more for historical reasons than for it being a very promising target. Coma is one of the best-studied clusters, and it hosts the prototype radio halo and relic (e.g., Thierbach et al. 2003; Brown \& Rudnick 2011). A HXR excess at the halo location was claimed by Rephaeli \& Gruber (2002) and Fusco-Femiano et al. (2004), but later never confirmed (Wik et al. 2009, 2011; Gastaldello et al. $2015)$. The radio relic $(1253+275)$ is located at $75^{\prime}$ from the cluster centre. Figure 4 shows the synchrotron and IC modelling for both the radio halo and relic.

For the relic, the current upper limit (Feretti \& Neumann 2006) appears more constraining than what is achievable with ASTRO-H HXI in $1 \mathrm{Ms}$ of observation according to the signalto-noise analysis. However, this upper limit is determined in the $20-80 \mathrm{keV}$ band and could be evaded with a low-energy spectral cutoff or flattening. Therefore, as for AS753, we also show the case with $\gamma_{\min }=1000$ in the right-hand panel of Fig. 4. Also in the case of the halo, the current HXR upper limit is at the same level as our predictions for $B_{\mathrm{dt}}$. This is due to our assumption of uniform radio sources, which is particularly inappropriate for objects with a large size in the sky, like Coma. In fact, in Tables 6 and 7, we try to assess this by providing alternative 
estimates by i) assuming that all the radio emission comes from a region within the ASTRO-H HXI FoV (we show this also for a few other objects); and ii) by computing the thermal ICM emission for the case of the halo only within the halo boundaries. (We normally integrate over the full halos size, but again, owing to the large sky size of Coma, here the difference is appreciable.)

Kitayama et al. (2014) have recently discussed the prospects for $500 \mathrm{ks}$ of ASTRO-H observation of the halo region. They estimate a corresponding 90\%CL upper limit of $B \sim 0.4 \mu \mathrm{G}$, which is slightly more optimistic than our estimates, but they assumed a photon index of $\alpha=2$.

\section{Conclusions}

In this work, we modelled the synchrotron and IC emission of all known radio halos and relics for which the spectral index can be determined. Our approach is phenomenological towards the generation mechanism of relativistic electrons. We simply assumed that the same electrons generating the observed radio synchrotron emission are responsible for HXR IC emission, where we adopted a power-law distribution with low- and high-energy cutoffs that we either fitted to the radio data, if steepening was observed, or we fixed to theoretically motivated values.

We provided updated lower limits on the magnetic field values for those objects for which an HXR upper limit is available. Subsequently, by considering the thermal ICM and CXB emissions and the instrumental background, we provided predictions for the volume-average magnetic filed values up to which HXR IC emission can be detected by the ASTRO-H HXI instrument, adopting different approaches and considering different observations times.

Our first approach was to estimate the magnetic field testable by ASTRO-H by taking the intersection point between the dominant background emission as reference, either thermal ICM or $\mathrm{CXB}$, and the properly scaled ASTRO-H sensitivity for $1 \mathrm{Ms}$ of observations. We then adopted a more robust approach based on photon counts in the $20-80 \mathrm{keV}$ regime, requiring the signalto-noise ratio to be higher than 5 , where the noise includes the ICM and CXB emissions and the instrumental background. With this approach, on which we have based our conclusions, we estimated the magnetic field values testable by ASTRO-H in $100 \mathrm{ks}$, $500 \mathrm{ks}$, and $1 \mathrm{Ms}$ of observations. With this estimate, we found that the observation time needed to test a certain magnetic field value $B$ roughly scales as $B \propto T_{\mathrm{obs}}^{1 /(2(1+\alpha))}$.

We identified as promising all those halos and relics for which the magnetic field testable by ASTRO-H in $100 \mathrm{ks}$ is larger than $1 \mu \mathrm{G}$ or than the equipartition estimates, where available. We provided the corresponding list of 15 halos and relics in Table 9. Among the most promising targets, we have the AS753 and A3667-NW relics and the A2255 and A2319 halos, with an extension in the sky close to or larger than the ASTRO-H HXI FoV. Additionally, we can also identify the A0085, A2048, and A2063 relics as very promising, and the A1914 halo, with an extension smaller than the HXI FoV. The size in the sky of the considered object plays a crucial role in such observations, both for evaluating the contribution of the thermal ICM emission to the observed spectrum and for planning multiple pointings with a corresponding increase in observation time.

We stress that we have provided a theoretical expectation that suffers from several approximations that we discuss openly in the text. While we estimate that most of these approximations imply conservative results, some of them could go in the other direction: in particular, the uncertainty in the ICM density distribution of some objects, particularly for radio relics in the clusters' outskirts, and the assumption of a power-law distribution of electrons down to low energies that are not tested by current radio observations, but could be tested soon by LOFAR. Therefore, while it is clear that detailed ASTRO-H observation simulations should be performed to select the best target amongst those we have proposed here, in particular against their angular dimension in the sky, we conclude that HXR IC detection could be at hand for ASTRO-H in several objects.

With the operation of NuSTAR and the upcoming launch of ASTRO-H, we will eventually be able to probe HXR IC emission in clusters for magnetic fields $\geq 1 \mu \mathrm{G}$. Although detailed observation simulation are needed case by case, for a few clusters we might finally break the degeneracy between the magnetic field and the relativistic electrons distribution, and be able to shed new light on the non-thermal phenomena in clusters of galaxies.

Acknowledgements. We thank the anonymous referee for helping to improve our manuscript. We thank Hiroki Akamatsu, Madoka Kawaharada, Matteo Murgia, Anders Pinzke, Jacco Vink, and Franco Vazza for useful comments and discussions. We also profited from the Radio Observation of Galaxy Clusters database, collected by Klaus Dolag. This work was supported by the Netherlands Organisation for Scientific Research (NWO) through a Veni and a Vidi grant (F.Z. and S.A.).

\section{References}

Ajello, M., Rebusco, P., Cappelluti, N., et al. 2009, ApJ, 690, 367 Ajello, M., Rebusco, P., Cappelluti, N., et al. 2010, ApJ, 725, 1688 Akamatsu, H., de Plaa, J., Kaastra, J., et al. 2012, PASJ, 64, 49

Bacchi, M., Feretti, L., Giovannini, G., \& Govoni, F. 2003, A\&A, 400, 465 Arnaud, K. A. 1996, in Astronomical Data Analysis Software and Systems V, eds. G. H. Jacoby, \& J. Barnes, ASP Conf. Ser., 101, 17

Bagchi, J., Enßlin, T. A., Miniati, F., et al. 2002, New Astron., 7, 249 Bagchi, J., Sirothia, S. K., Werner, N., et al. 2011, ApJ, 736, L8 Barrena, R., Girardi, M., Boschin, W., \& Dasi, M. 2009, A\&A, 503, 357 Beck, R., \& Krause, M. 2005, Astron. Nach., 326, 414

Blumenthal, G. R., \& Gould, R. J. 1970, Rev. Mod. Phys., 42, 237 Böhringer, H., \& Werner, N. 2010, A\&ARv, 18, 127

Bonafede, A., Feretti, L., Giovannini, G., et al. 2009a, A\&A, 503, 707 Bonafede, A., Giovannini, G., Feretti, L., Govoni, F., \& Murgia, M. 2009b, A\&A, 494, 429

Bonafede, A., Feretti, L., Murgia, M., et al. 2010, A\&A, 513, A30

Bonafede, A., Brüggen, M., van Weeren, R., et al. 2012, MNRAS, 426, 40

Bonafede, A., Vazza, F., Brüggen, M., et al. 2013, MNRAS, 433, 3208

Boschin, W., Girardi, M., \& Barrena, R. 2013, MNRAS, 434, 772

Brentjens, M., A. 2008, A\&A, 489, 69

Brown, S., \& Rudnick, L. 2011, MNRAS, 412, 2

Brown, S., Duesterhoeft, J., \& Rudnick, L. 2011, ApJ, 727, L25

Brunetti, G. 2003, in Matter and Energy in Clusters of Galaxies, eds. S. Bowyer, \& C.-Y. Hwang, ASP Conf. Ser., 301, 349

Brunetti, G., \& Jones, T. W. 2014, Int. J. Mod. Phys. D, 23, 30007

Brunetti, G., Setti, G., \& Comastri, A. 1997, A\&A, 325, 898

Brunetti, G., Giacintucci, S., Cassano, R., et al. 2008, Nature, 455, 944

Brunetti, G., Rudnick, L., Cassano, R., et al. 2013, A\&A, 558, A52

Carilli, C. L., \& Taylor, G. B. 2002, ARA\&A, 40, 319

Cavagnolo, K. W., Donahue, M., Voit, G. M., \& Sun, M. 2009, ApJ, 182, 12

Cavaliere, A., \& Fusco-Femiano, R. 1976, A\&A, 49, 137

Chen, Y., Reiprich, T. H., Böhringer, H., Ikebe, Y., \& Zhang, Y. Y. 2007, A\&A, 466, 805

Chen, C. M. H., Harris, D. E., Harrison, F. A., \& Mao, P. H. 2008, MNRAS, 383, 1259

Clarke, T. E. 2004, J. Kor. Astron. Soc., 37, 337

Clarke, T. E., \& Ensslin, T. A. 2006, AJ, 131, 2900

Cohen, A. S., \& Clarke, T. E. 2011, AJ, 141, 149

Comastri, A., Setti, G., Zamorani, G., \& Hasinger, G. 1995, A\&A, 296, 1

Eckert, D., Produit, N., Paltani, S., Neronov, A., \& Courvoisier, T. J.-L. 2008, A\&A, 479, 27

Enßlin, T. A. 2002, A\&A, 396, L17

Enßlin, T. A., \& Vogt, C. 2006, A\&A, 453, 447

Ensslin, T. A., \& Sunyaev, R. A. 2002, A\&A, 383, 423

Ensslin, T. A., Biermann, P. L., Klein, U., \& Kohle, S. 1998, A\&A, 332, 395 
Feretti, L., \& Neumann, D. M. 2006, A\&A, 450, L21

Feretti, L., Boehringer, H., Giovannini, G., \& Neumann, D. 1997a, A\&A, 317, 432

Feretti, L., Giovannini, G., \& Boehringer, H. 1997b, New Astron., 2, 501

Feretti, L., Fusco-Femiano, R., Giovannini, G., \& Govoni, F. 2001, A\&A, 373, 106

Feretti, L., Orru, E., Brunetti, G., et al. 2004, A\&A, 423, 111

Feretti, L., Schuecker, P., Böhringer, H., Govoni, F., \& Giovannini, G. 2005, A\&A, 444, 157

Feretti, L., Giovannini, G., Govoni, F., \& Murgia, M. 2012, A\&ARv, 20, 1

Finoguenov, A., Sarazin, C. L., Nakazawa, K., Wik, D. R., \& Clarke, T. E. 2010, ApJ, 715, 1143

Fukazawa, Y., Makishima, K., \& Ohashi, T. 2004, PASJ, 56, 965

Fusco-Femiano, R., Orlandini, M., Brunetti, G., et al. 2004, ApJ, 602, L73

Gastaldello, F., Wik, D. R., Molendi, S., et al. 2015, ApJ, 800, 139

Gavazzi, G., \& Trinchieri, G. 1983, ApJ, 270, 410

Giacintucci, S. 2011, Mem. Soc. Astron. It., 82, 541

Giacintucci, S., Venturi, T., Brunetti, G., et al. 2005, A\&A, 440, 867

Giacintucci, S., Venturi, T., Brunetti, G., et al. 2009, A\&A, 505, 45

Giacintucci, S., Dallacasa, D., Venturi, T., et al. 2011, A\&A, 534, A109

Giacintucci, S., Kale, R., Wik, D. R., Venturi, T., \& Markevitch, M. 2013, ApJ, 766,18

Gilli, R., Comastri, A., \& Hasinger, G. 2007, A\&A, 463, 79

Giovannini, G., \& Feretti, L. 2000, New Astron., 5, 335

Giovannini, G., Feretti, L., \& Stanghellini, C. 1991, A\&A, 252, 528

Giovannini, G., Bonafede, A., Feretti, L., et al. 2009, A\&A, 507, 1257

Govoni, F., \& Feretti, L. 2004, Int. J. Mod. Phys. D, 13, 1549

Govoni, F., Feretti, L., Giovannini, G., et al. 2001, A\&A, 376, 803

Govoni, F., Murgia, M., Feretti, L., et al. 2005, A\&A, 430, L5

Gruber, D. E. 1992, in The X-ray Background, eds. X. Barcons, \& A. C. Fabian, 44

Harrison, F. A., Craig, W. W., Christensen, F. E., et al. 2013, ApJ, 770, 103

Henriksen, M., \& Mushotzky, R. 2001, ApJ, 553, 84

Hindson, L., Johnston-Hollitt, M., Hurley-Walker, N., et al. 2014, MNRAS, 445, 330

Johnston-Hollitt, M. 2004, in The Riddle of Cooling Flows in Galaxies and Clusters of galaxies, eds. T. Reiprich, J. Kempner, \& N. Soker, 51

Juett, A. M., Sarazin, C. L., Clarke, T. E., et al. 2008, ApJ, 672, 138

Kale, R., \& Dwarakanath, K. S. 2012, ApJ, 744, 46

Kale, R., Dwarakanath, K. S., Bagchi, J., \& Paul, S. 2012, MNRAS, 426, 1204

Kassim, N. E., Clarke, T. E., Enßlin, T. A., Cohen, A. S., \& Neumann, D. M. 2001, ApJ, 559, 785

Kawano, N., Fukazawa, Y., Nishino, S., et al. 2009, PASJ, 61, 377

Kempner, J. C., \& Sarazin, C. L. 2001, ApJ, 548, 639

Kitayama, T., Bautz, M., Markevitch, M., et al. 2014, ArXiv e-prints [arXiv: 1412.1176]

Komissarov, S. S., \& Gubanov, A. G. 1994, A\&A, 285, 27

Liang, H., Hunstead, R. W., Birkinshaw, M., \& Andreani, P. 2000, ApJ, 544, 686

Lindner, R. R., Baker, A. J., Hughes, J. P., et al. 2014, ApJ, 786, 49

Longair, M. 2011, High Energy Astrophysics (Cambridge University Press)

Ma, C.-J., Ebeling, H., Donovan, D., \& Barrett, E. 2008, ApJ, 684, 160

Macario, G., Venturi, T., Intema, H. T., et al. 2013, A\&A, 551, A11

Mantz, A., Allen, S. W., Ebeling, H., Rapetti, D., \& Drlica-Wagner, A. 2010, MNRAS, 406, 1773

Marini, F., Bardelli, S., Zucca, E., et al. 2004, MNRAS, 353, 1219

Menanteau, F., Hughes, J. P., Sifón, C., et al. 2012, ApJ, 748, 7

Million, E. T. \& Allen, S. W. 2009, MNRAS, 399, 1307

Molendi, S., \& Gastaldello, F. 2009, A\&A, 493, 13

Mori, H., Maeda, Y., Ishida, M., et al. 2012, in SPIE Conf. Ser., 8443, 5

Murgia, M., Govoni, F., Feretti, L., et al. 2004, A\&A, 424, 429

Murgia, M., Eckert, D., Govoni, F., et al. 2010, A\&A, 514, A76

Nakazawa, K., Sarazin, C. L., Kawaharada, M., et al. 2009, PASJ, 61, 339

Ogrean, G. A., Brüggen, M., van Weeren, R. J., et al. 2013, MNRAS, 433, 812

Orru, E., Murgia, M., Feretti, L., et al. 2007, A\&A, 467, 943

Ota, N. 2012, RA\&A, 12, 973

Ota, N., Nagayoshi, K., Pratt, G. W., et al. 2014, A\&A, 562, A60

Pacholczyk, A. 1970, Radio Astrophysics: Nonthermal Processes in Galactic and Extragalactic Sources, Astronomy and Astrophysics Series (W. H. Freeman) Pandey-Pommier, M., Richard, J., Combes, F., et al. 2013, A\&A, 557, A117
Pérez-Torres, M. A., Zandanel, F., Guerrero, M. A., et al. 2009, MNRAS, 396, 2237

Petrosian, V. 2001, ApJ, 557, 560

Petrosian, V., Madejski, G., \& Luli, K. 2006, ApJ, 652, 948

Piffaretti, R., Arnaud, M., Pratt, G. W., Pointecouteau, E., \& Melin, J. B. 2011, A\&A, 534, A109

Pinzke, A., Pfrommer, C., \& Bergström, L. 2011, Phys. Rev. D, 84, 123509

Pizzo, R., F., \& de Bruyn, A., G. 2009, A\&A, 507, 639

Planck Collaboration XXIX. 2014, A\&A, 571, A29

Popesso, P., Biviano, A., Böhringer, H., \& Romaniello, M. 2007, A\&A, 461, 397

Reid, A. D., Hunstead, R. W., Lémonon, L., \& Pierre, M. M. 1999, MNRAS, 302, 571

Rephaeli, Y. 1979, ApJ, 227, 364

Rephaeli, Y., \& Gruber, D. 2002, ApJ, 579, 587

Rephaeli, Y., Gruber, D., \& Arieli, Y. 2006, ApJ, 649, 673

Rephaeli, Y., Nevalainen, J., Ohashi, T., \& Bykov, A. M. 2008, Space Sci. Rev., 134,71

Röttgering, H., Afonso, J., Barthel, P., et al. 2012, JA\&A, 557, 34

Röttgering, H., van Weeren, R., Brüggen, M., et al. 2013, Astron. Nachr., 334, 333

Rottgering, H. J. A., Wieringa, M. H., Hunstead, R. W., \& Ekers, R. D. 1997, MNRAS, 290, 577

Sarazin, C. 1999, ApJ, 520, 529

Sarazin, C. L. 1988, X-ray emission from clusters of galaxies (Cambridge: Cambridge University Press)

Sarazin, C. L., \& Lieu, R. 1998, ApJ, 494, L177

Sehgal, N., Hughes, J. P., Wittman, D., et al. 2008, ApJ, 673, 163

Shen, S., Kauffmann, G., Von Der Linden, A., White, S. D. M., \& Best, P. N. 2008, MNRAS, 389, 1074

Shimwell, T. W., Brown, S., Feain, I. J., et al. 2014, MNRAS, 440, 2901

Slee, O. B., Roy, A. L., Murgia, M., Andernach, H., \& Ehle, M. 2001, AJ, 122, 1172

Smith, R. K., Brickhouse, N. S., Liedahl, D. A., \& Raymond, J. C. 2001, ApJ, 556, L91

Storm, E., Jeltema, T. E., \& Rudnick, L. 2015, MNRAS, 448, 2495

Stroe, A., Rumsey, C., Harwood, J. J., et al. 2014, MNRAS, 441, 41

Subrahmanyan, R., Beasley, A. J., Goss, W. M., Golap, K., \& Hunstead, R. W. 2003, AJ, 125, 1095

Takahashi, T., Mitsuda, K., Kelley, R., et al. 2010, in SPIE Conf. Ser., 7732

Takahashi, T., Mitsuda, K., Kelley, R., et al. 2012, in SPIE Conf. Ser., 84431

Takahashi, T., Mitsuda, K., Kelley, R., et al. 2014, in SPIE Conf. Ser., 9144, 25

Thierbach, M., Klein, U., \& Wielebinski, R. 2003, A\&A, 397, 53

Turner, M. J. L., Abbey, A., Arnaud, M., et al. 2001, A\&A, 365, L27

Vacca, V., Feretti, L., Giovannini, G., et al. 2014, A\&A, 561, A11

van Weeren, R. J., Röttgering, H. J. A., Bagchi, J., et al. 2009, A\&A, 506, 1083

van Weeren, R. J., Brüggen, M., Röttgering, H. J. A., et al. 2011a, A\&A, 533, A35

van Weeren, R. J., Hoeft, M., Röttgering, H. J. A., et al. 2011b, A\&A, 528, A38 van Weeren, R. J., Röttgering, H. J. A., \& Brüggen, M. 2011c, A\&A, 527, A114 van Weeren, R. J., Bonafede, A., Ebeling, H., et al. 2012a, MNRAS, 425, L36

van Weeren, R. J., Röttgering, H. J. A., Intema, H. T., et al. 2012b, A\&A, 546, A 124

van Weeren, R. J., Röttgering, H. J. A., Rafferty, D. A., et al. 2012c, A\&A, 543, A43

van Weeren, R. J., Fogarty, K., Jones, C., et al. 2013, ApJ, 769, 101

Venturi, T., Bardelli, S., Dallacasa, D., et al. 2003, A\&A, 402, 913

Venturi, T., Giacintucci, S., Brunetti, G., et al. 2007, A\&A, 463, 937

Venturi, T., Giacintucci, S., Cassano, R., et al. 2009, in The Low-Frequency Radio Universe, eds. D. J. Saikia, D. A. Green, Y. Gupta, \& T. Venturi, ASP Conf. Ser., 407, 232

Vogt, C., \& Enßlin, T. A. 2005, A\&A, 434, 67

Voit, G. M. 2005, Rev. Mod. Phys., 77, 207

Wik, D. R., Sarazin, C. L., Finoguenov, A., et al. 2009, ApJ, 696, 1700

Wik, D. R., Sarazin, C. L., Finoguenov, A., et al. 2011, ApJ, 727, 119

Wik, D. R., Sarazin, C. L., Zhang, Y.-Y., et al. 2012, ApJ, 748, 67

Wik, D. R., Hornstrup, A., Molendi, S., et al. 2014, ApJ, 792, 48

Yoon, J. H., Schawinski, K., Sheen, Y.-K., Ree, C. H., \& Yi, S. K. 2008, ApJS, 176,414

Zandanel, F., Pfrommer, C., \& Prada, F. 2014, MNRAS, 438, 116 


\section{Appendix A: Other figures and comments on individual clusters}

This appendix contains the figures for the synchrotron and IC modelling of all clusters not shown in the main text. For some of these, the IC emission peaks in SXRs or EUV above the CXB and ICM emissions, so we also show the case with $\gamma_{\min }=1000$.

Several objects, mainly radio relics, have the peculiarity of very low thermal ICM emission, probably an underestimation by observations pointed to the cluster's centre or by using the phenomenological model of Zandanel et al. (2014) for the gas density. In some cases (A0781, A2163, MACSJ1149, 5-2223-E and W,
MACSJ1752.0+4440-SW, PLCK G287.0+32.9-N and S), the CXB outweighs the thermal ICM emission, and in addition, the intersection point between the ASTRO-H and CXB emissions is at an energy lower than the rise in the CXB spectrum. The corresponding results for $B_{\mathrm{dt}}$ should be taken with caution, and in fact, in these cases, the signal-to-noise approach gives more conservative and reasonable results.

We also remind the reader that the signal-to-noise approach assumes a straight power law in the $20-80 \mathrm{keV}$ energy band (see Sect. 7). While in most cases the cutoffs are at much lower and higher energies, for A2063 and A0013 this method could give slightly optimistic results.

All figures can be read as explained in the caption of Fig. 2. 
A\&A 582, A20 (2015)
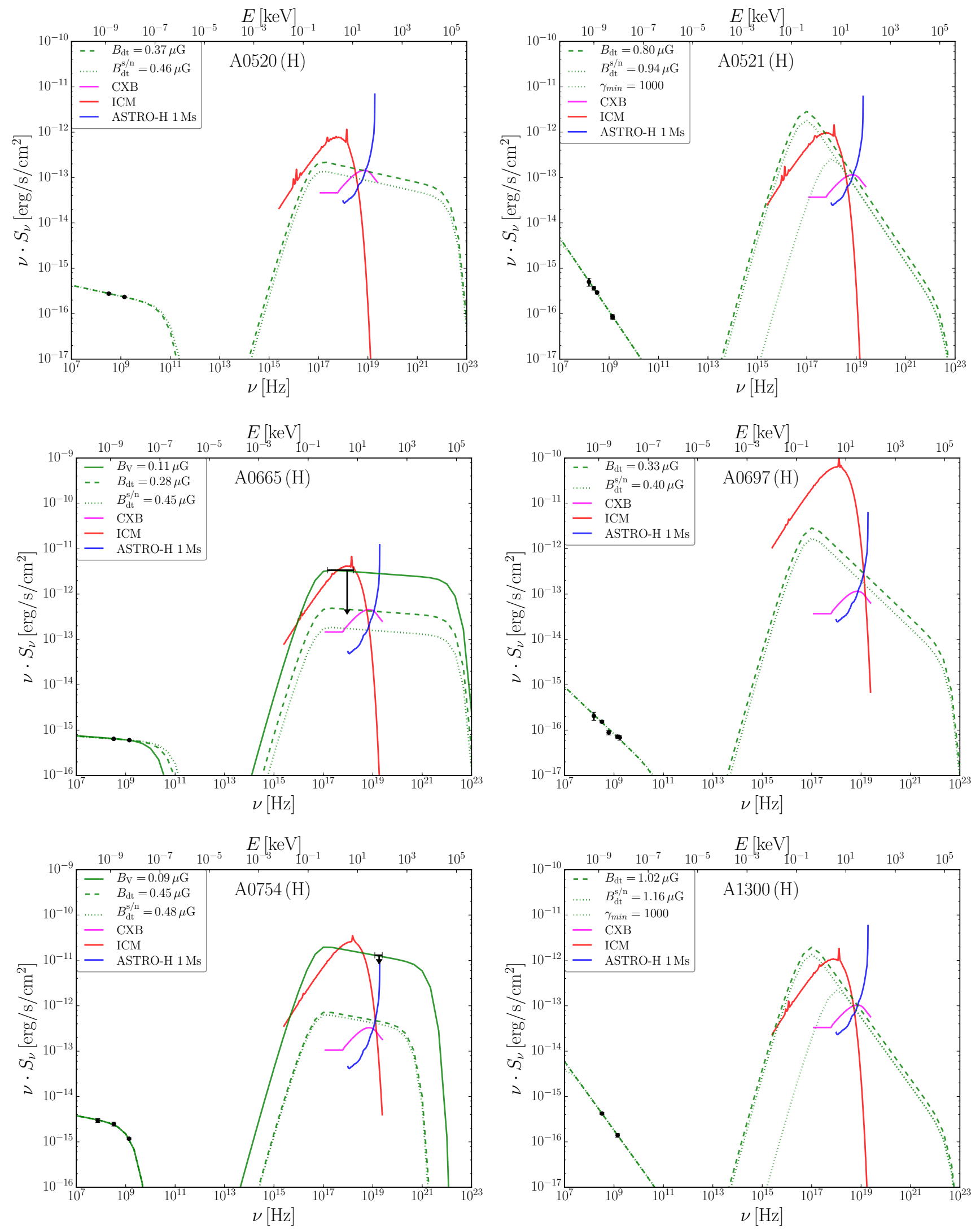

Fig. A.1. Synchrotron and IC modelling for all clusters that are not shown in the main text. Caption as in Fig. 2. 
R. Bartels et al.: Inverse-Compton emission from clusters of galaxies
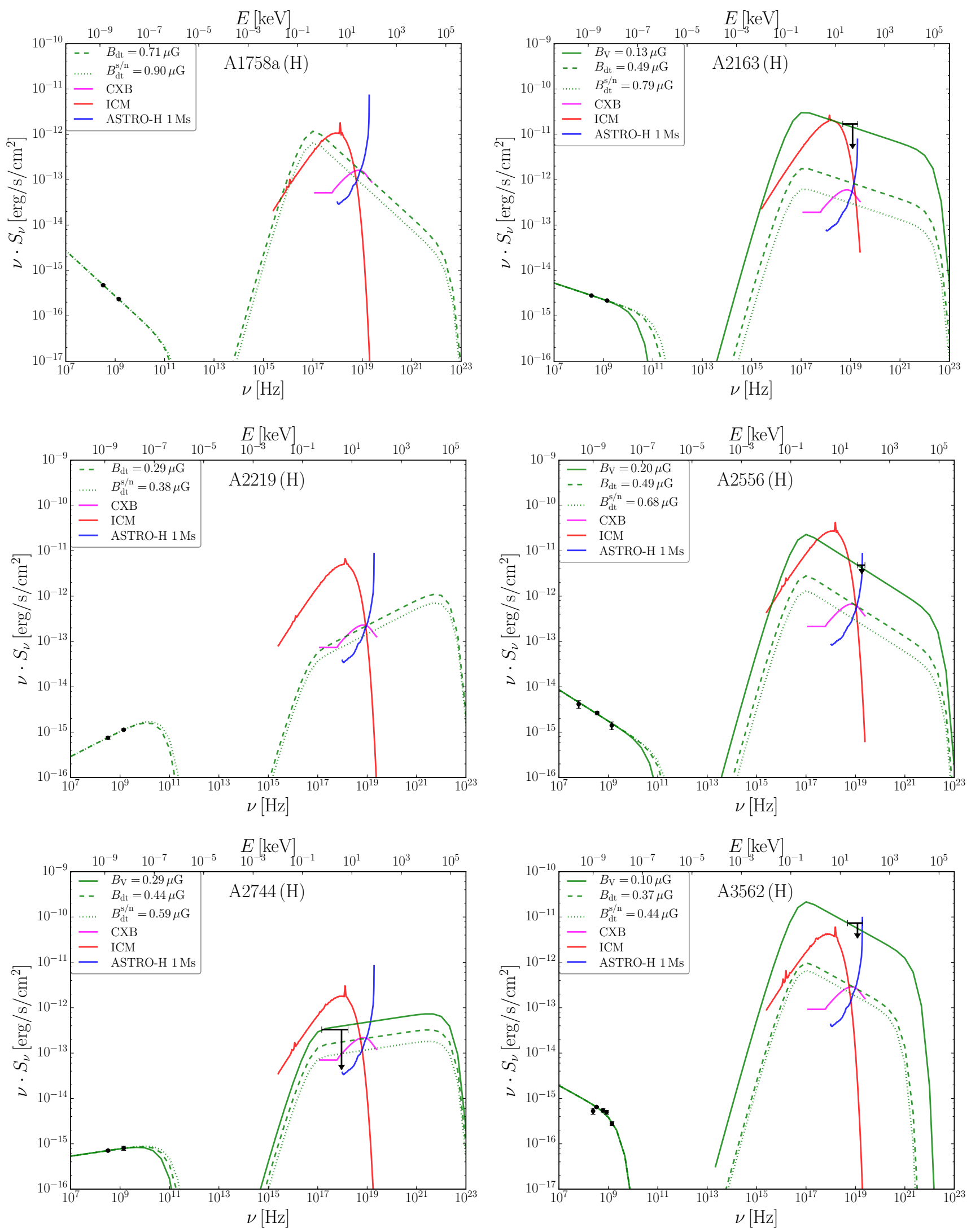

Fig. A.1. continued.

A20, page 19 of 27 
A\&A 582, A20 (2015)
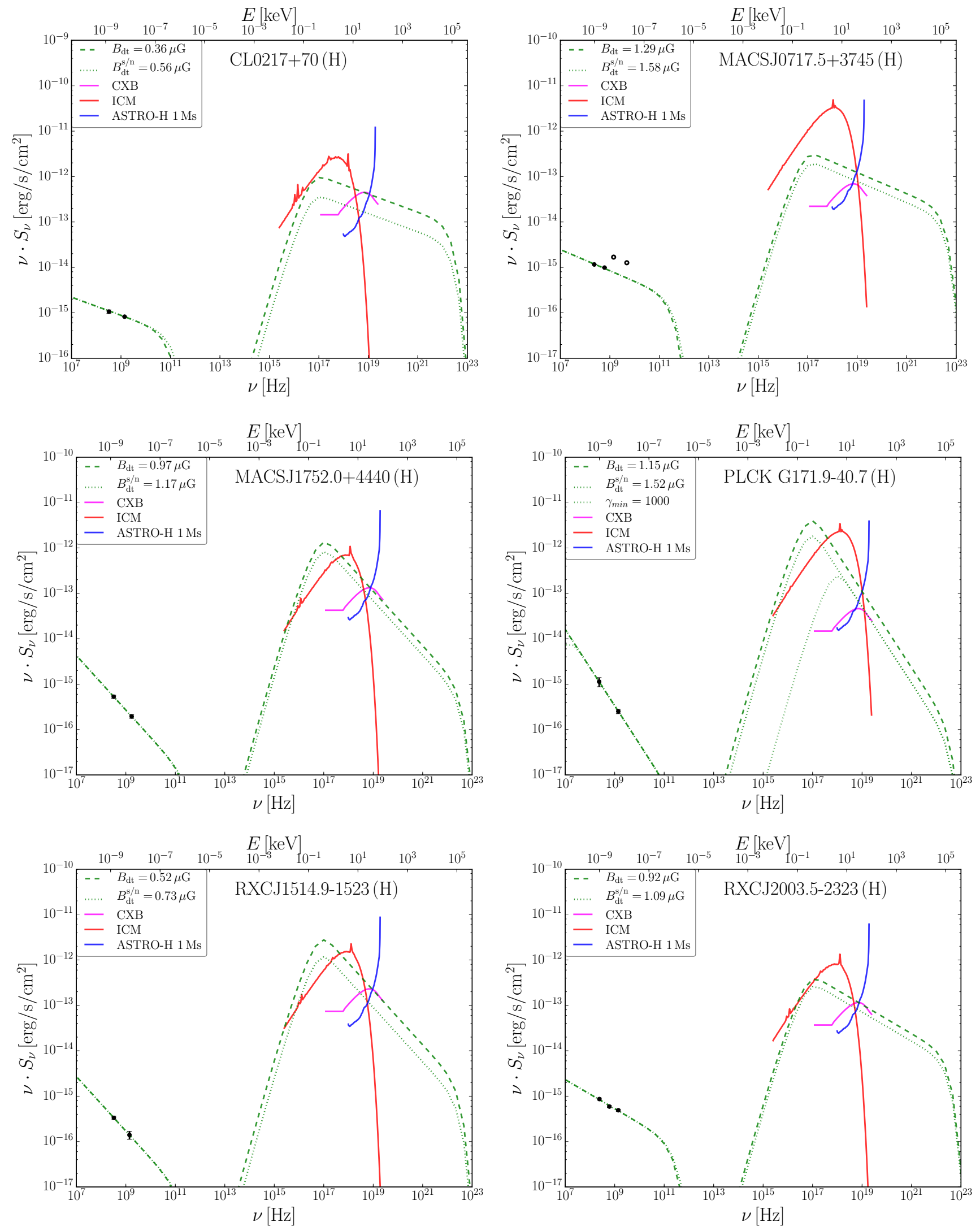

Fig. A.1. continued. 
R. Bartels et al.: Inverse-Compton emission from clusters of galaxies
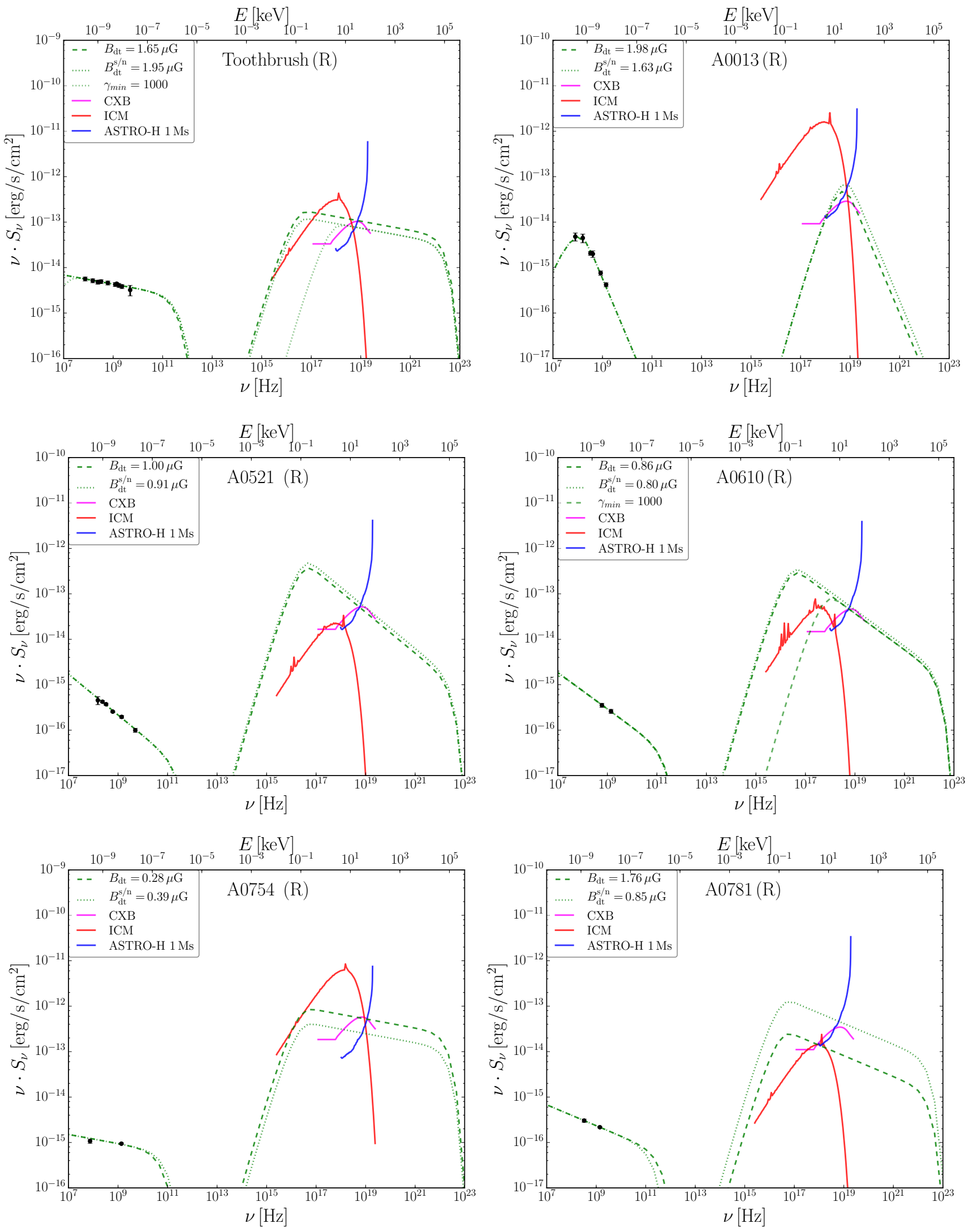

Fig. A.1. continued. 
A\&A 582, A20 (2015)
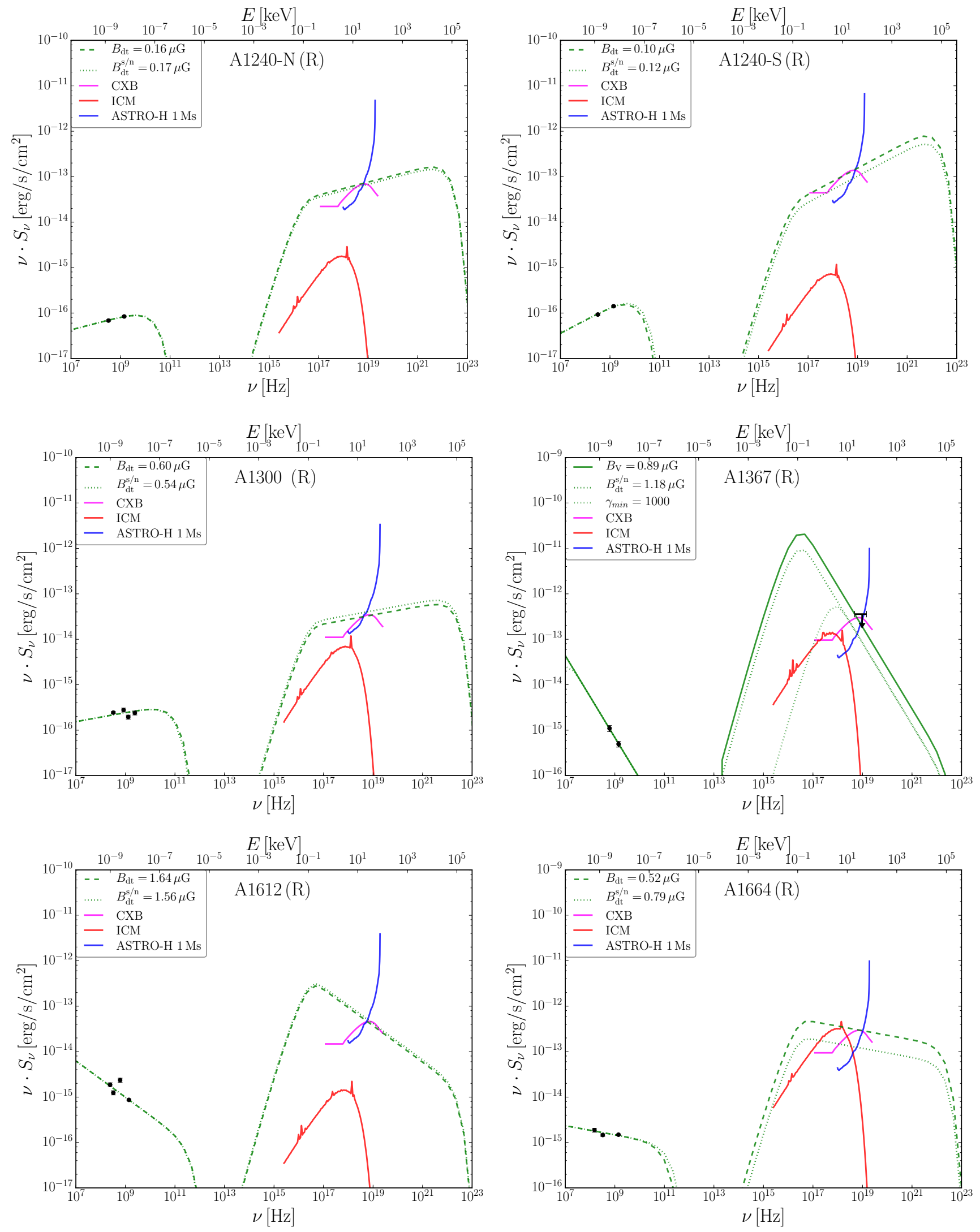

Fig. A.1. continued. 
R. Bartels et al.: Inverse-Compton emission from clusters of galaxies
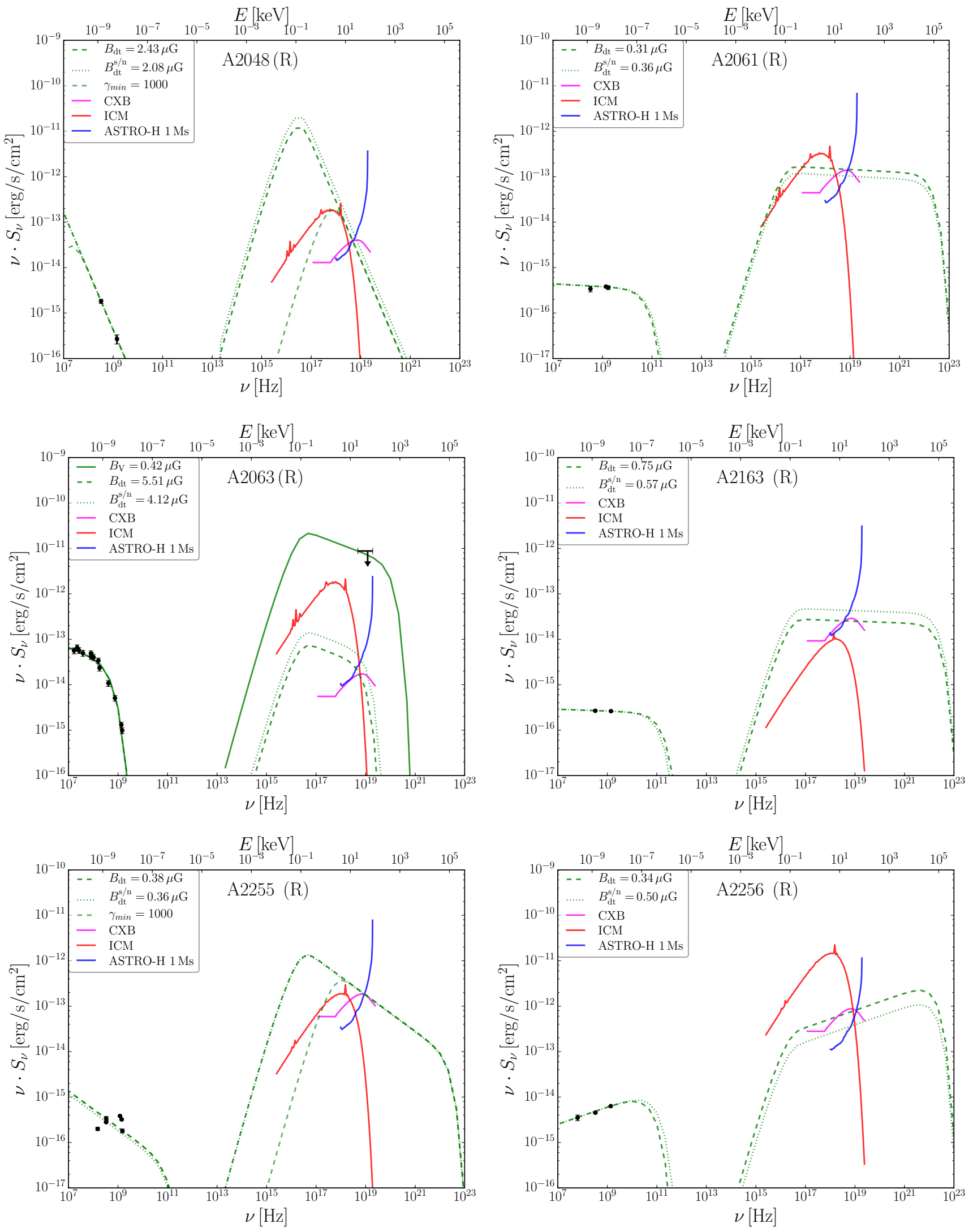

Fig. A.1. continued. 
A\&A 582, A20 (2015)
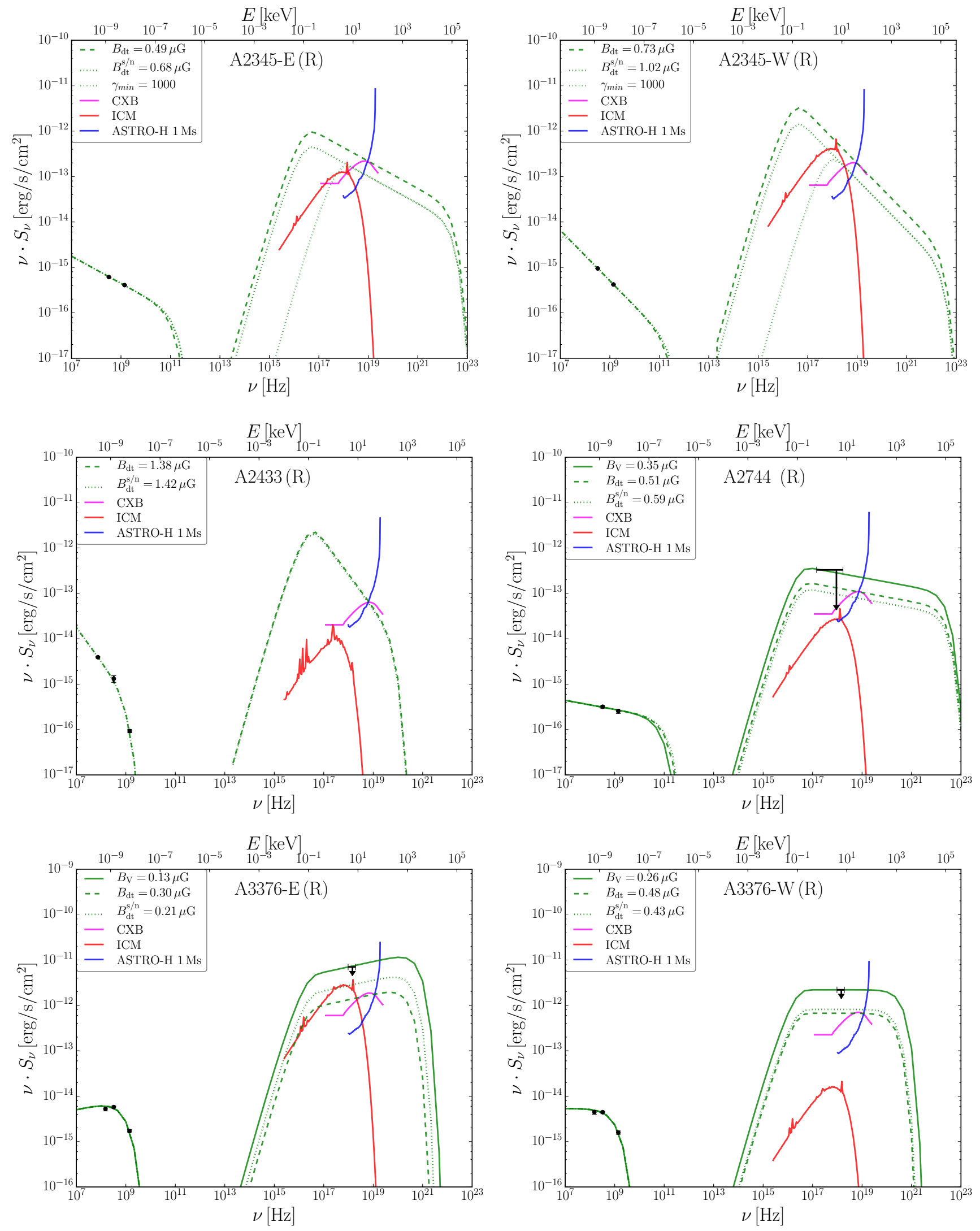

Fig. A.1. continued. 
R. Bartels et al.: Inverse-Compton emission from clusters of galaxies
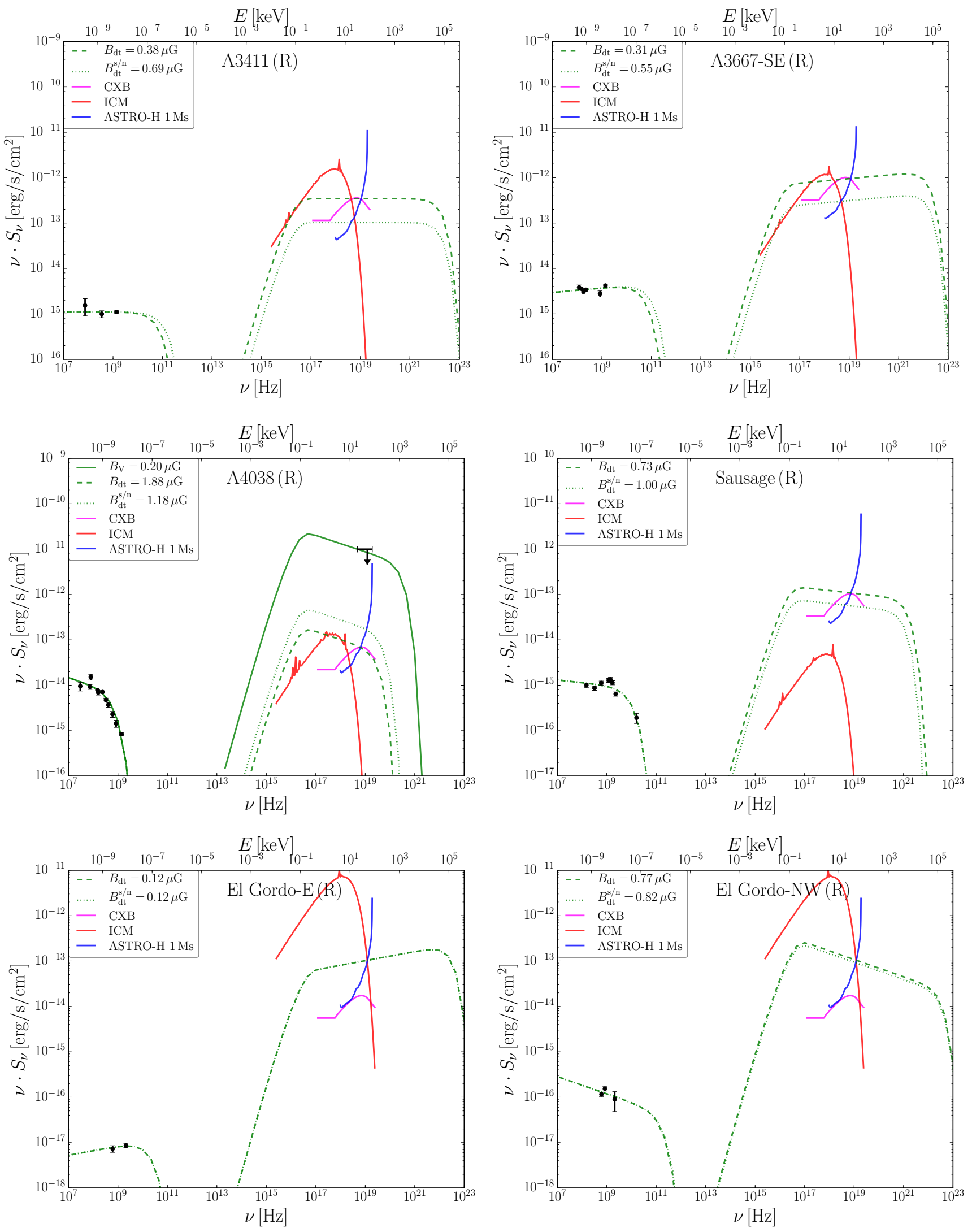

Fig. A.1. continued. 
A\&A 582, A20 (2015)
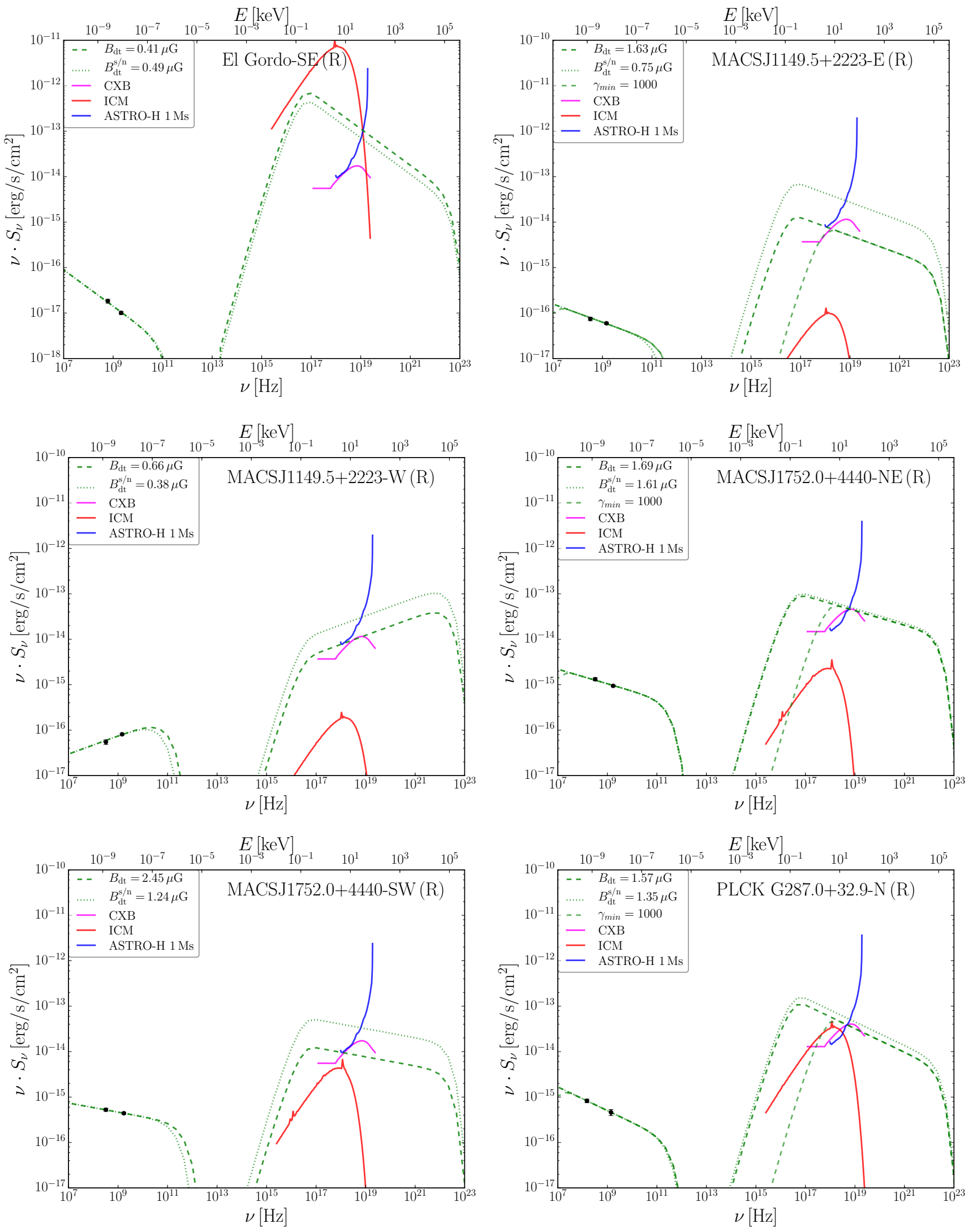

Fig. A.1. continued. 
R. Bartels et al.: Inverse-Compton emission from clusters of galaxies
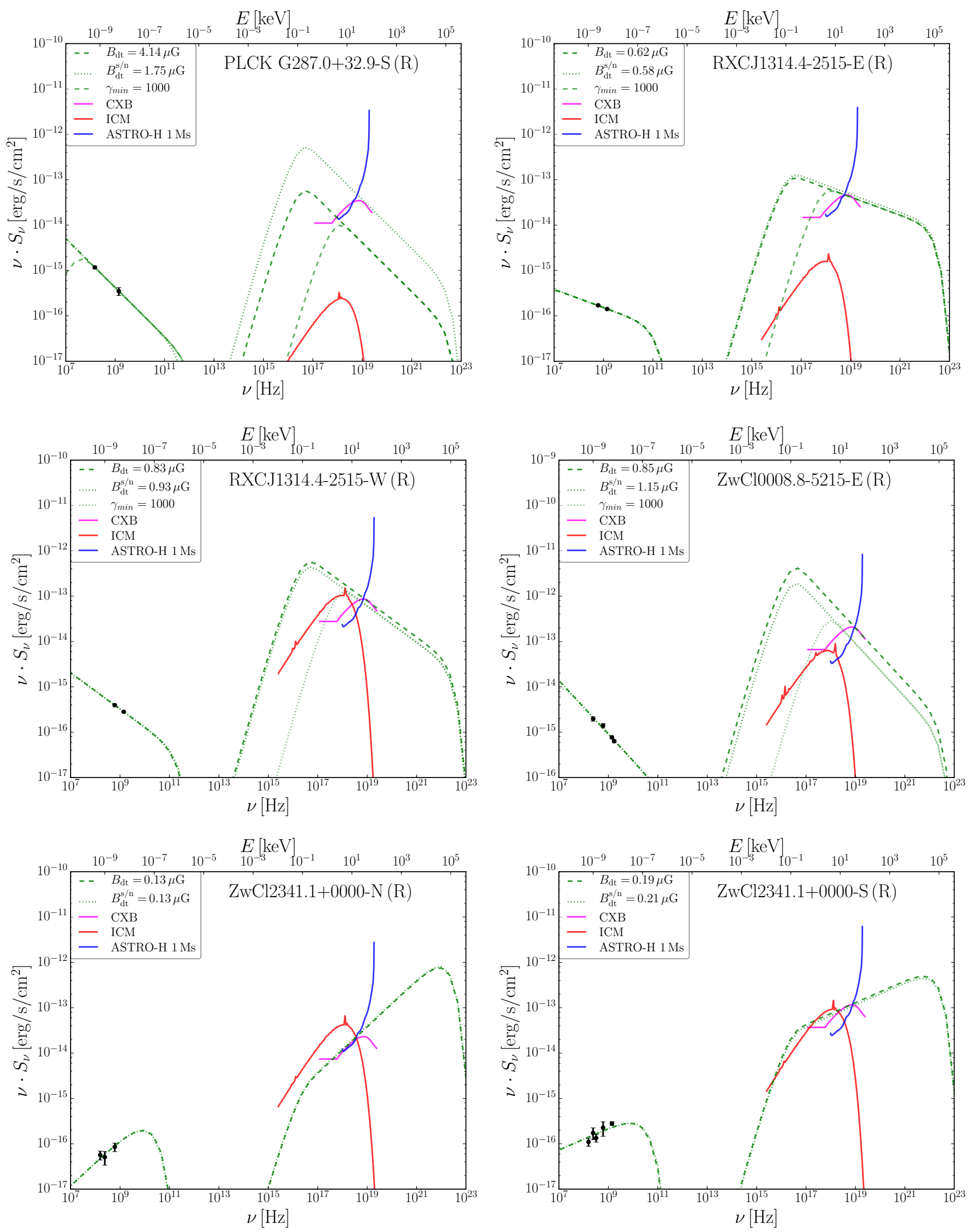

Fig. A.1. continued.

A20, page 27 of 27 